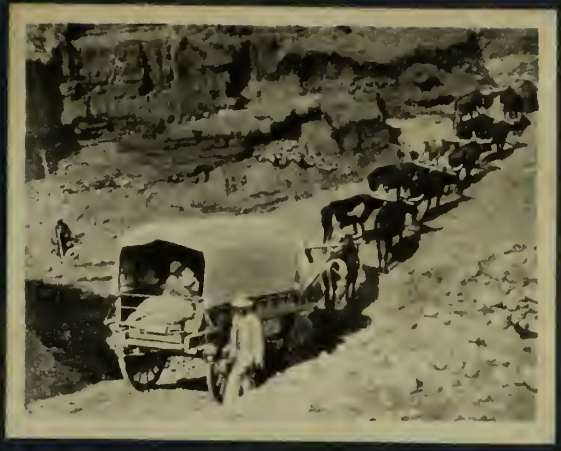




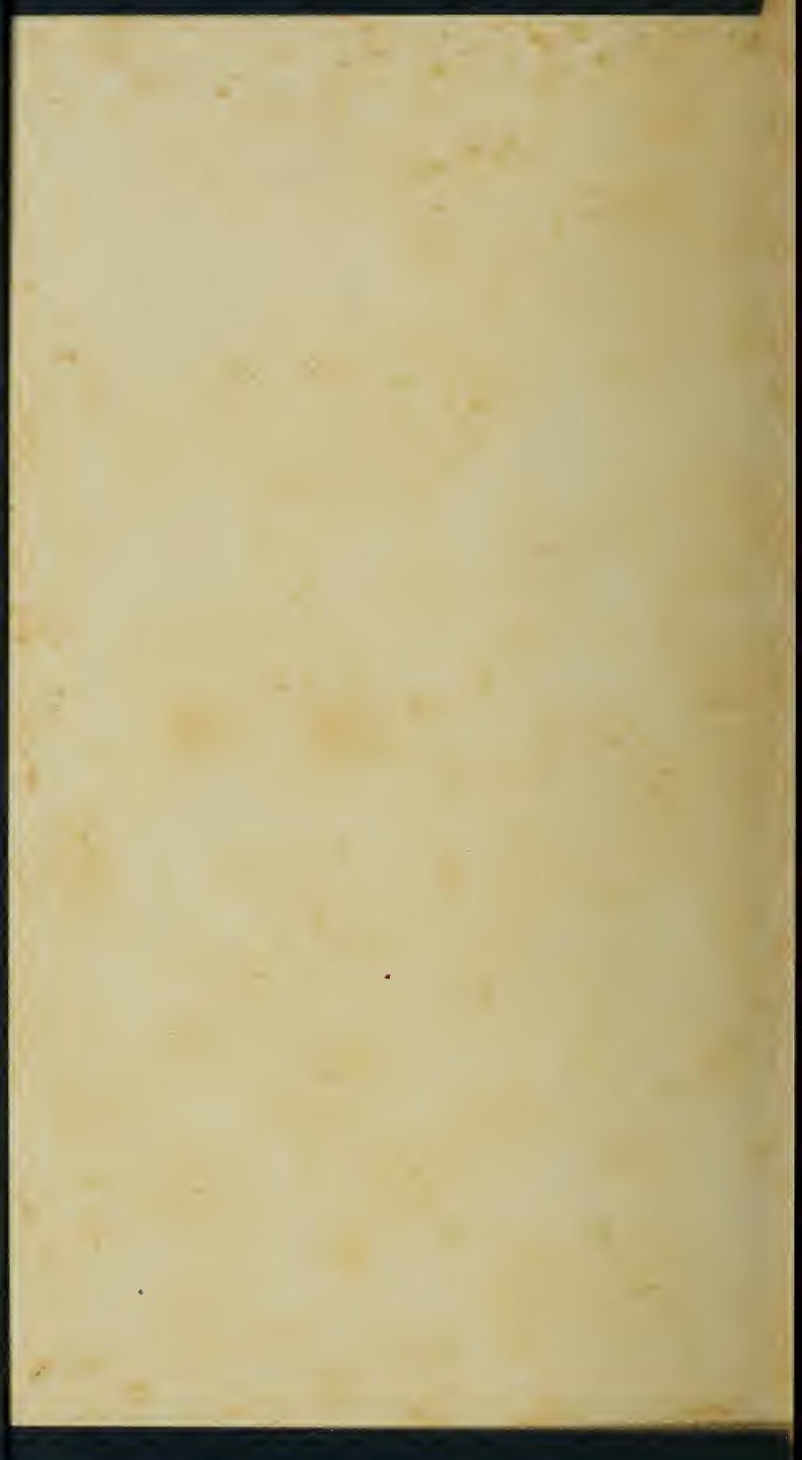


99925 aft.

Qver lears bous sonstenus

Oelped Bentrand.

Alice Bertraind.

DANS LE SUD-AFRICAIN

ET AU SEUIL DE L'AFRIQUE CENTRALE

Geneve. Iannier 1911. 



\section{Dans le/}

\section{SUD-AFRIC A I N \\ et au seuil de l'Afrique Centrale}

PAR

\section{ALFRED BERTRAND//}

Ancien Président de la Société de Géographie de Genève

Membre Honoraire et Correspondant

de la Société Royale de Géographie de Londres,

de la Société Royale de Géographie Écossaise, etc.

58 Illustrations d'après les Photographies de l'Auteur

\section{$\mathbb{S B}$}

GENÈVE

Librairie J. H. JEHEBER

Rue du Marché, 28
PARIS

Librairie FISCHBACHER

33, Rue de Seine 

Au Pays des ba-Souto, la Suisse de l'Afrique Australe. Le Jubilé de Morija. - Visites aux stations missionnaires. - Dans l'East Griqualand. - Par les montagnes, traversée à cheval du Pays des ba-Souto, de l'est à l'ouest. Quelques données géographiques sur le Basoutoland. L'Orange et ses affluents. - La chute de la Malétsunyané. - Le "Zambezi Express».

Pays des ba-Rotsi, au seuil de l'Afrique centrale. - Les chutes Victoria du Haut-Zambèze et la ligne ferrée du Cap au Caire. - La station missionnaire et le nouveau "Settlement de Livingstone".

Transformations récentes au pays des ba-Rotsi. Quelques réflexions sur l'œurre missionnaire. La nouvelle Confédération des Etats du Sud-Africain. 
NB. - L'auteur doit à l'obligeance de la Direction des Chemins de fer du Cap les cinq photographies suivantes: Arrivée au Cap - Jeune Matébélé - Coup-d'œil sur la ville du Cap - Dans le parc de "Groote-Shur" - Le Cap de Bonne-Espérance.

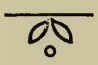




\section{DANS LE SUD AFRICAIN}

Nous nous sommes embarqués le 26 septembre $1908, \mathrm{M}^{\mathrm{me}}$ Bertrand et moi, à bord de "l'Armadale Castle ", l'un des meilleurs steamers de la flotte de "l'Union Castle Line ", compagnie qui fait le service de la poste en reliant Southampton au Cap. Nous nous rendions, en qualité de délégués indépendants, à la célébration du Jubilé qui devait commémorer le soixante-quinzième anniversaire de la mission au pays des ba-Souto ${ }^{1}$, cette contrée afri-

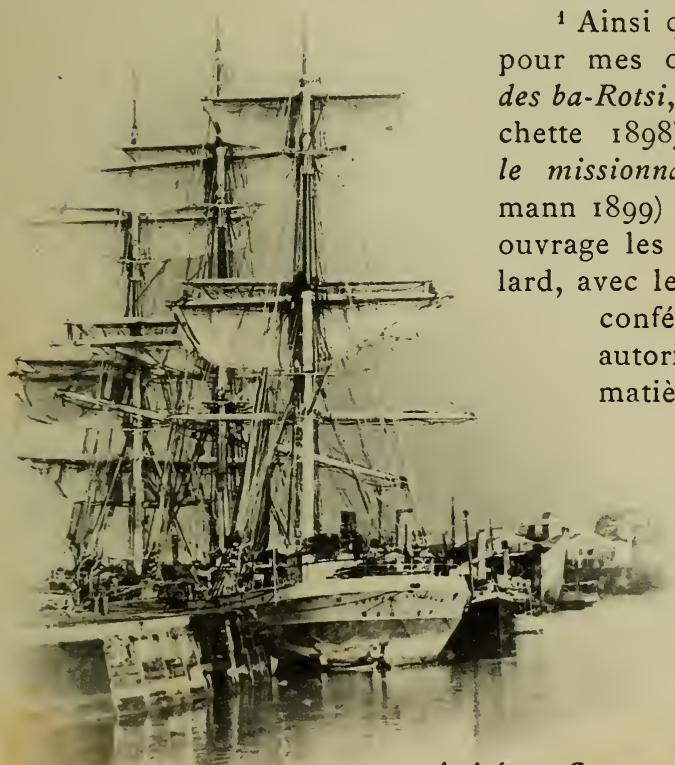

Arrivée au Cap années en Afrique, - avait posées concernant l'orthographe 


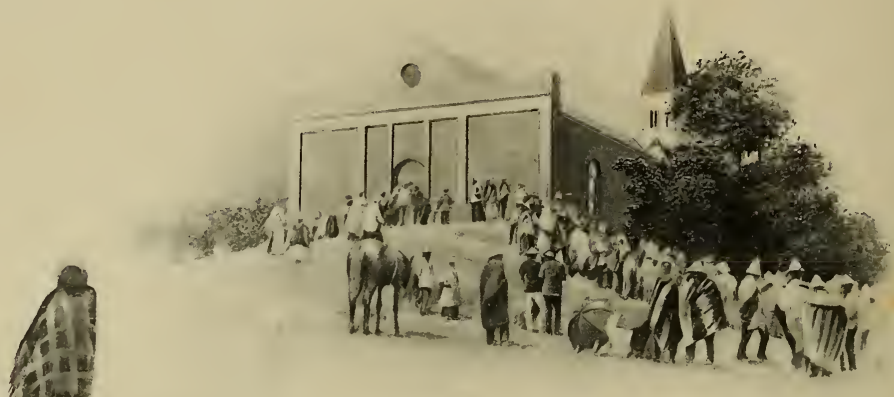

L'Eglise de Morija

caine montagneuse, appelée la “ Suisse de l'AfriqueAustrale ".

Après avoir navigué à travers l'Océan Atlantique

française des noms de tribus ou peuplades qui se trouvent dans le pays des ba-Souto, le pays des ma-Tébélé, le pays des ba-Rotsi.

Les préfixes "ba " ou "ma" indiquent le pluriel. Ces préfixes représentent en quelque sorte l'article et prennent une minuscule tandis que le nom propre est écrit lui-même avec une majuscule.

Le préfixe "mo s'emploie au singulier pour indiquer un individu, exemple : un mo-Souto. Si l'on veut désigner la langue du pays, on emploie le préfixe "sé ", exemple: le sé-Souto. Quant aux noms de pays, plusieurs sont déjà anglaisés ou francisés; exemples: le Basoutoland, le Matébéléland, etc. Pour désigner le Pays des ba-Souto, j'emploie aussi le nom de Basoutoland pour désigner l'ensemble du pays et de Lessouto pour désigner le Haut-Plateau.

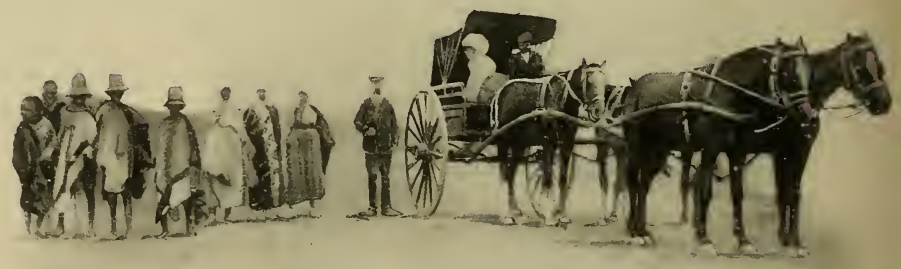

En route pour Morija 
sur un parcours de dix mille kilomètres, nous entrons, le I 3 octobre, dans le beau port duCap, cette ville si bien située au pied de la montagne de la Table, d'où le chemin de fer nous a amenés en deux nuits et une journée à Blœmfontein, la capitale de l'Etat d'Orange. De là nous atteignons en quelques heures la frontière ouest du Basoutoland et Maséru, la résidence du Gouverneur.

Grâce à l'excellente organisation des fêtes du Jubilé, nous avons trouvé à Maséru des "carts", voitures haut perchées sur deux roues et attelées de quatre chevaux; c'est le moyen de locomotion qui, avec le cheval de selle, est le plus usité dans ce pays où les routes n'existent qu'à l'état rudimentaire.

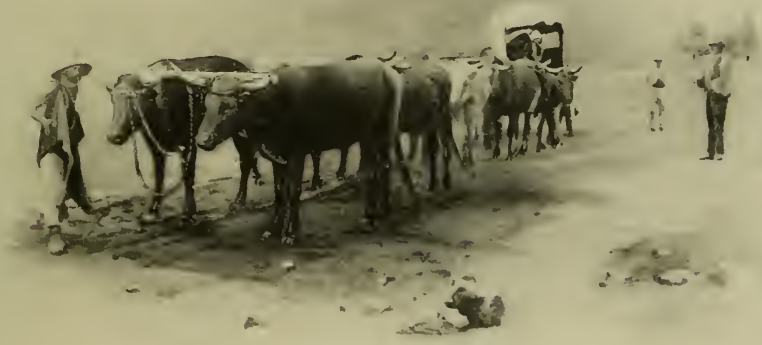

Autre moyen de transport

C'est ainsi qu'en une journée nous arrivons, avec nombre d'autres délégués, à Morija, où nous recevons des missionnaires l'accueil le plus cordial. Sur la route suivie, la population chrétienne des villages environnants et les enfants des écoles, drapeaux 
déployés, viennent à notre rencontre en chantant.

Le 2 I octobre igo8, Morija est en fête. Le temps est magnifique, et partout les drapeaux flottent gaiement au vent. Des milliers d'indigènes, revêtus d'étoffes aux couleurs voyantes, arrivent à Morija qui à pied, qui à cheval, qui en chariots attelés de longues files de bœufs. Ici et là s'improvisent des campements pittoresques autour desquels paissent chevaux et bestiaux. A neuf heures, une grande foule s'installe sur l'herbe ombragée par des eucalyptus, près de l'église qui aurait été beaucoup trop petite pour contenir cette foule. Sur une estrade prennent place les délégués de la Société des Missions évangéliques de $\mathrm{Pa}$ ris : MM. les pasteurs Bianquis et Dumas, ainsi que M. Bonzon, pasteur à Pau, qui représente les églises de France. Le gouverneur du Basoutoland, M. Slowley, occupe la place d'honneur, et, près de lui, en costume européen, voici Letsie, le grand

M. Adolphe Jalla et le prince Litia 


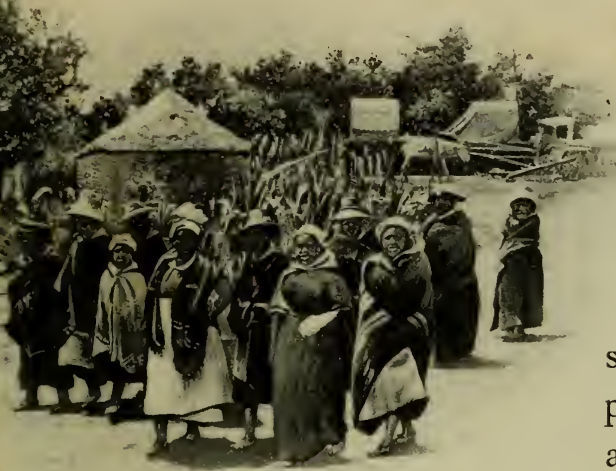

chef des baSouto. Chacun remarque la personnalité sympathique du prince Litia, qui a été envoyé par Indigènes se rendant au Jubilé Léwanika, le roi des ba-Rotsi, pour le représenter aux fêtes du Jubilé; le prince Litia est accompagné de deux des missionnaires du Haut-Zambèze, MM. Adolphe Jalla et Boiteux. Non loin d'eux, M. John Moffat, le beau-frère de Livingstone; puis, le vénérable missionnaire Creux, de Prétoria, et $\mathbf{M}$. Jacques, de Laurenzo-Marquès qui représentent la mission de la Suisse romande; on sait les liens intimes qui existent entre cette mission et celle du pays des ba-Souto.

Il y avait là des délégués représentant seize sociétés de missions européennes et américaines qui travaillent au sud de l'Afrique, sans parler de dix ou douze grands chefs et de soixante délégués des églises indigènes.

Sous la présidence de M. Edouard Jacottet, de nombreuses allocutions ont été prononcées et lecture a été donnée d'un message très flatteur dans lequel le gouverneur de l'Afrique du Sud, Lord Selbourne, exprimait à la Société des Missions évangéliques de Paris toute sa reconnaissance pour le bien qu'elle 
a fait au cours de ces soixante-quinze années. De touchantes paroles ont été prononcées à la mémoire du regretté François Coillard, " le père, le conseiller, le médiateur entre les tribus sauvages et tous les missionnaires qui ont travaillé dans les régions du Haut Zambèze ». La vénérable $\mathrm{M}^{\mathrm{me}}$ Adolphe Mabille, la doyenne des missionnaires, en quelques paroles énergiques, s'est adressée directement aux chefs ba-Souto qui l'entouraient.

Les délégués des Sociétés de missions du Sud de l'Afrique ont rendu hommage, non seulement à l'œuvre de civilisation chrétienne accomplie chez les ba-Souto, mais aussi à l'influence qu'exerce cette mission sur les autres missions, grâce à son organisation ecclésiastique et à son union intime avec la nation des ba-Souto et le gouvernement. Bref, elle a résolu le problème difficile de la fondation d'une église générale au pays des ba-Souto.

A la fin de la première journée, un banquet joyeux et fraternel a réuni les délégués et autres blancs dans la nouvelle et spacieuse salle de l'école normale, tandis que M. Louis Mabille présidait à la distribution de la nourriture de plusieurs milliers d'indigènes, chrétiens et païens, qu'avaient attirés les fètes du Jubilé.

Ce n'était certes pas une tâche facile pour les missionnaires de Morija, dans une contrée où il n'est pas toujours aisé de s'approvisionner, que de donner largement l'hospitalité à quatre-vingts blancs et de nourrir d'innombrables ba-Souto. Pour qui connaît 


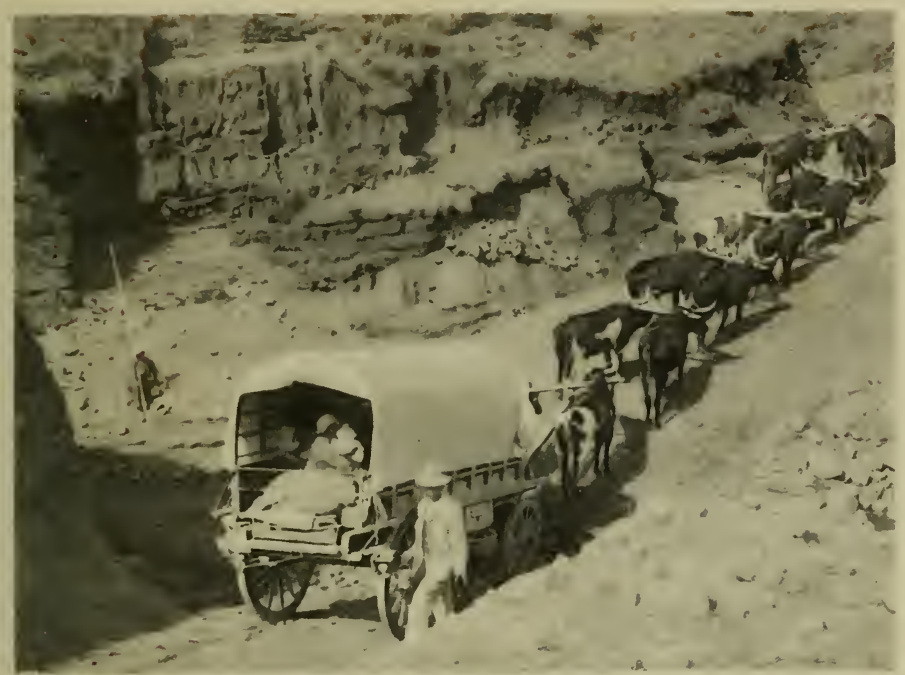

En route pour Thabana-Morena

les foules païennes laissées à elles-mêmes, chacun aurait été frappé, preuve vivante de l'influence bienfaisante qu'exerce la mission, de la tranquillité, de la discipline et de la décence parfaites de ces nombreux indigènes.

Elle est grande l'œuvre de civilisation chrétienne accomplie au cours de ces soixante-quinze dernières années, sous les auspices de la Société des Missions évangéliques de Paris, dans ce pays des ba-Souto! C'est en r 833 que les pionniers, MM. Arbousset, Cassalis et Gosselin, fondèrent la station de Morija, le premier des postes missionnaires de la région. Puis, sous l'énergique impulsion de $M$. Adolphe Mabille, un vaudois, qui y travailla depuis 1859 
jusqu'en I 894, et celle de ses successeurs, cette région se couvrit d'annexes et d'écoles. Morija devint le centre spirituel de ce pays des ba-Souto où règnaient jadis le cannibalisme et toutes les horreurs du paganisme.

Il est juste de dire que la Suisse a joué un grand rôle dans cette œuvre de civilisation chrétienne; aujourd'hui, plus de la moitié des missionnaires qui y travaillent sont nos compatriotes.

Actuellement, aux quatorze stations principales, à la tête desquelles se trouvent des européens, et aux treize autres stations dirigées par des pasteurs indigènes, se rattachent deux cent dix annexes et deux cent vingt-huit écoles qui couvrent le pays du nord au sud et de l'est à l'ouest comme d'un vaste filet dont les mailles se resserrent tous les jours davantage. On trouve dans ces écoles onze mille jeunes ba-Souto des deux sexes qui sont instruits et éduqués par deux cent quatre-vingt-quinze instituteurs indigènes.

En outre, Morija possède une école de théologie

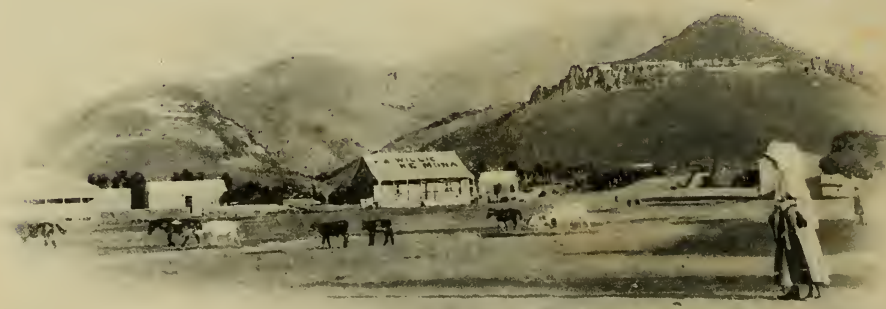

A Mohalesttok 
Sur les bords de l'Orange

dirigée par M. Edouard Jacottet, de Neuchâtel, école qui a déjà formé treize pasteurs indigènes actuellement en charge, et qui, pour le présent semestre, compte sept élèves.

M. R.-H. Dyke est à la tête d'une école normale, qui a fourni des centaines d'élèves dispersés dans tout le sud de l'Afrique. Les élèves qui ont terminé le cycle des études obtiennent le brevet d'instituteurs; plusieurs d'entre eux ont des emplois dans le gouvernement. L'école normale de Morija se place au premier rang des institutions indigènes du SudAfricain.

L'école biblique de Morija, que dirige aujourd'hui un genevois, M. Duby, a pour but de former des évangélistes. Cette institution exerce une bienfaisante influence et nombre de ses élèves travaillent, soit 
I 8

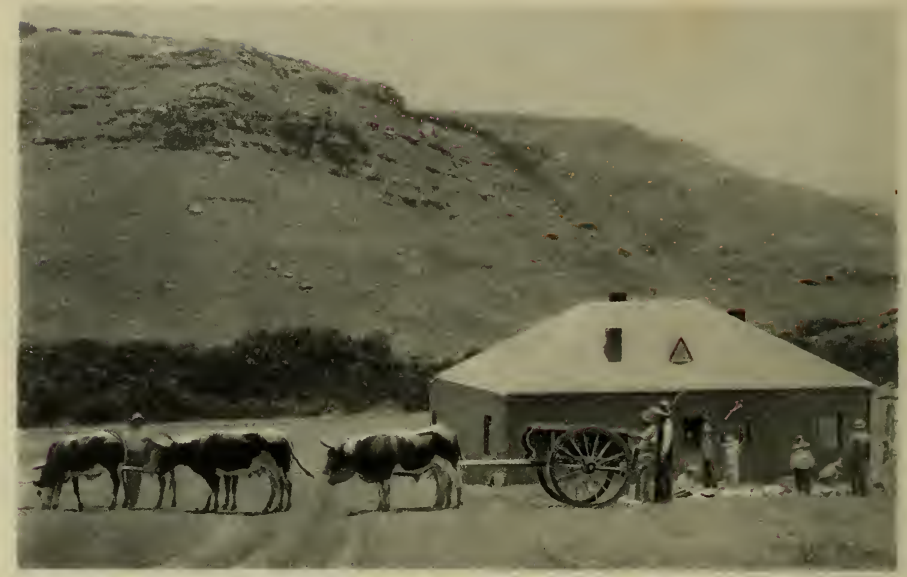

A l'Ecole Industrielle de Léloaleng

dans le pays des ba-Souto même, soit dans les contrées environnantes.

En outre, M. Duby a la direction du service de librairie qui s'est grandement développé depuis que

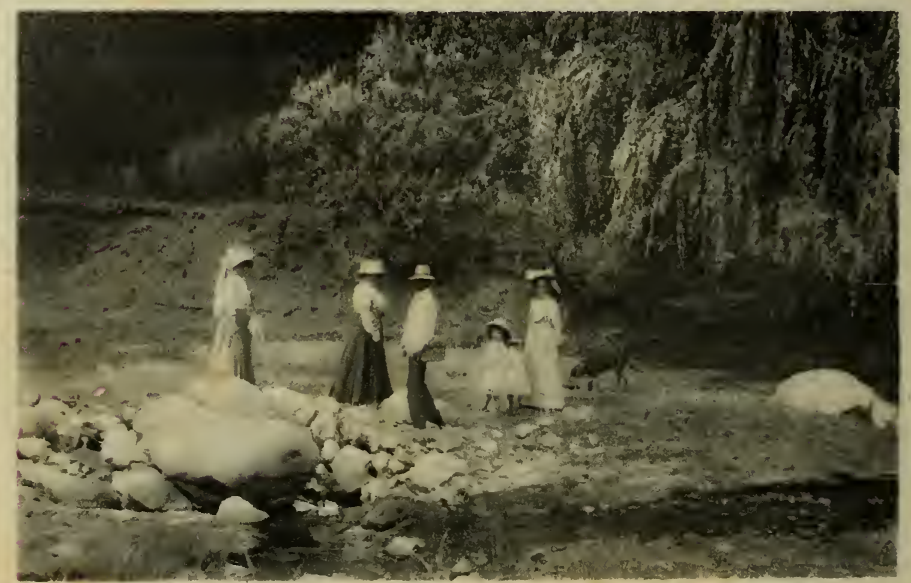

Aux environs de Quthing 


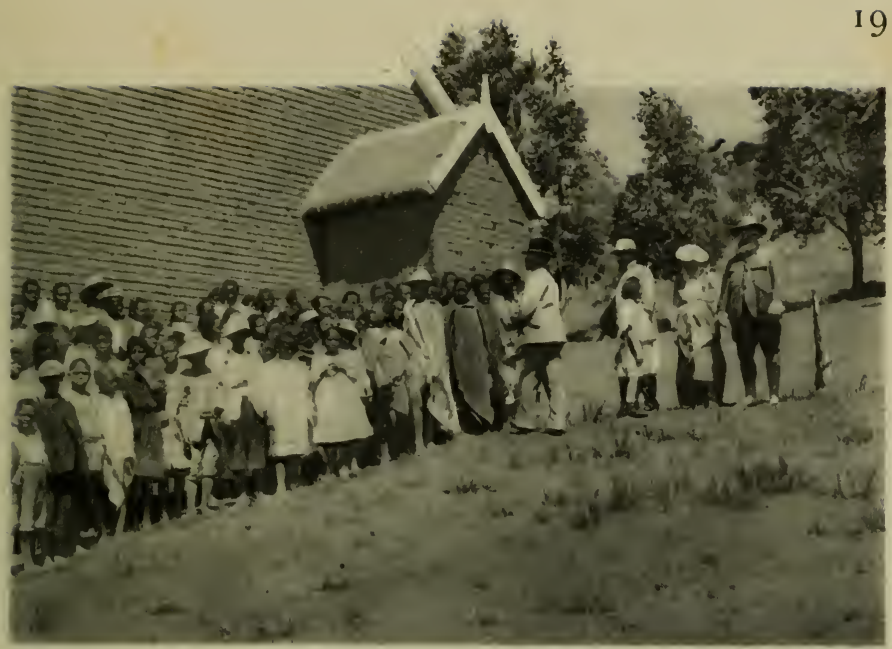

Enfants de l'Ecole missionnaire de Masitisi

l'imprimerie a été reprise, avec un outillage nouveau, par M. Labarthe de Genève.

N'oublions pas que les missionnaires ont posé les règles de la syntaxe et de la grammaire sé-souto et

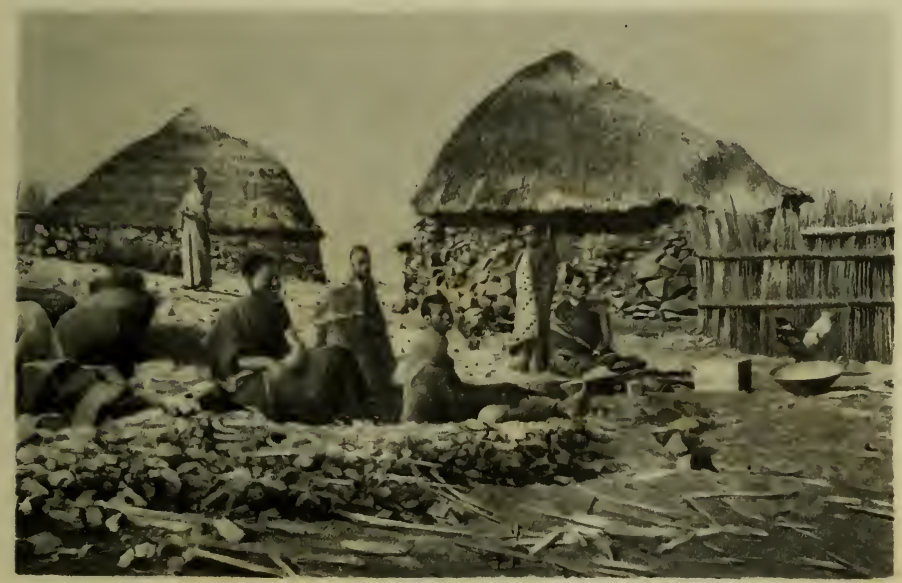

Village indigene 


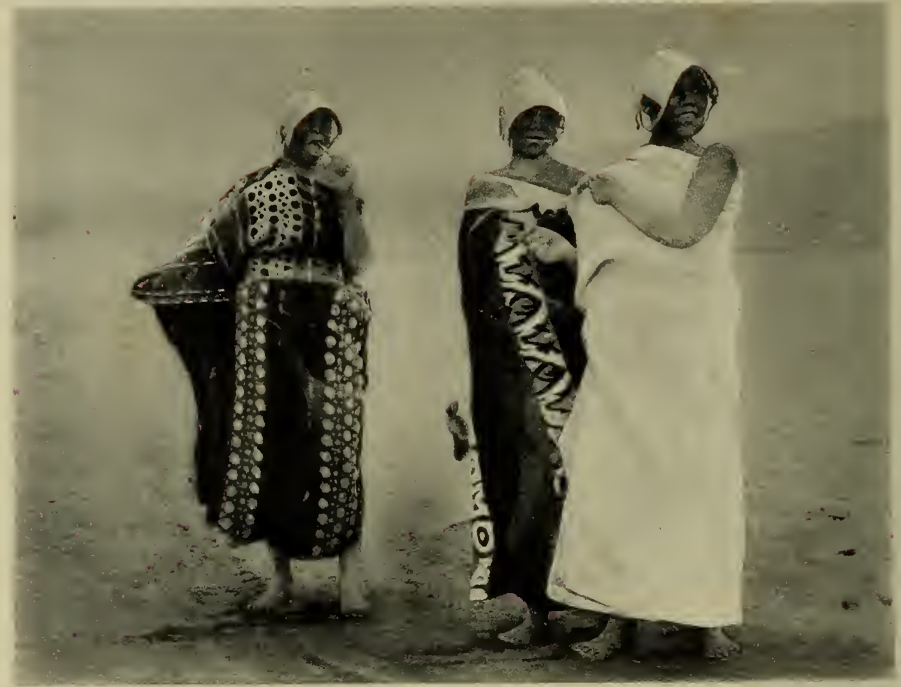

Jeunes filles "Bale“ sur la route d'Hermon

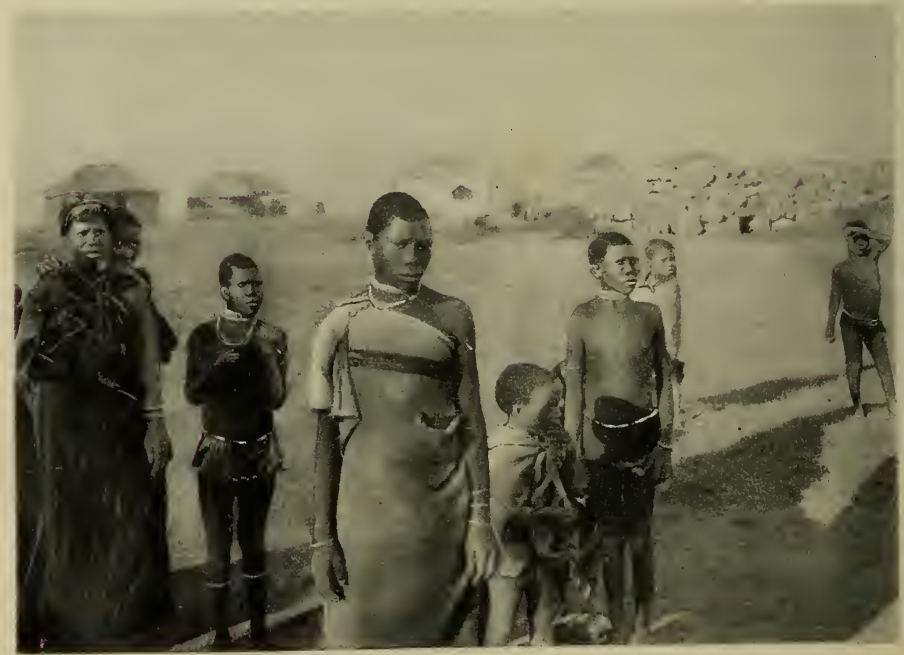

Près de Bethesda 
que l'influence de sa littérature s'étend sur le Transvaal, l'Etat d'Orange, le Béchuanaland et jusqu'audelà du Zambèze.

Nous avons aussi appris à connaître M. le $\mathrm{D}^{\mathrm{r}}$ Hertig et sa femme établis à Morija, des amis de l'œuvre missionnaire.

Une fois les fêtes du Jubilé terminées, nous avons

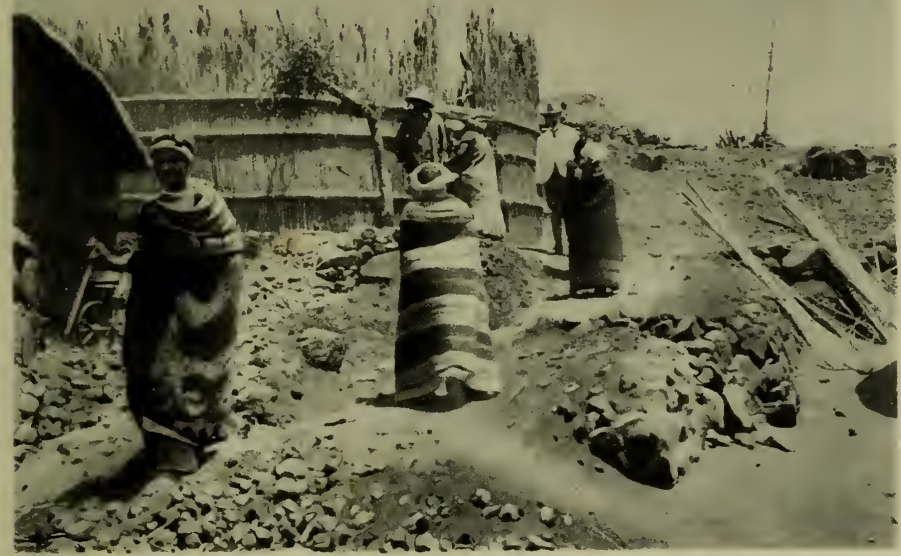

Près de Thaba-Bosiou

fait, $\mathrm{M}^{\mathrm{me}}$ Bertrand et moi, un fort intéressant voyage dans le sud et le centre du Basoutoland, visitant plusieurs stations missionnaires.

Comme je l'ai déjà dit, dans ce pays, le moyen de locomotion le plus rapide, est le cheval de selle ou le "cart ", une voiture à deux roues, haut juchée et attelée de chevaux plus ou moins bien dressés. On traverse de cette manière cols et plateaux, vallées 
et ravins dont les rivières sont passées à gué. Nous avons visité les stations missionnaires du sud et du centre du Basoutoland en commençant par ThabanaMorena que M. Paul Germond a habitée pendant longtemps et que dirigent aujourd'hui ses enfants M. et $M^{\text {me }}$ Louis Germond; cette station possède aussi une école supérieure pour les jeunes filles indigènes.

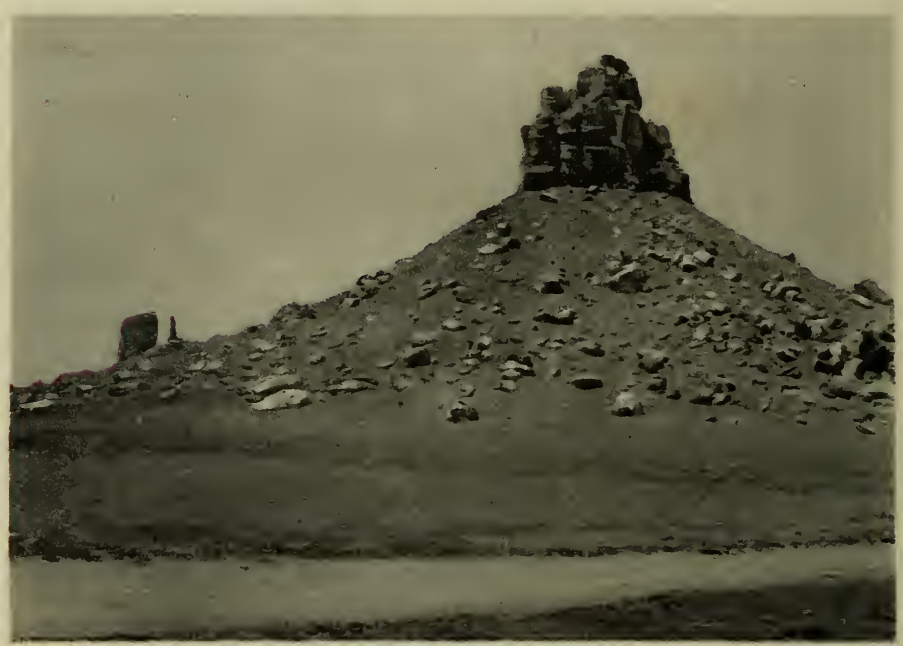

La montagne de Quiloané

En passant par Mohales Hook où nous nous arrêtons chez le représentant du gouvernement anglais, nous passons plusieurs jours à la station de Léloaleng située au pied du pittoresque camp de Quthing. Léloaleng est le siège de l'école industrielle pour jeunes gens ba-Souto, à la tête de laquelle se trouvent $\mathbf{M}$. et $\mathbf{M}^{\mathrm{me}}$ Verdier qui sont aidés par $\mathbf{M}$. et $\mathbf{M}^{\mathrm{me}} \mathbf{M a r t i n}$. Non loin de là, nous visitons Masitisi l'ancienne 


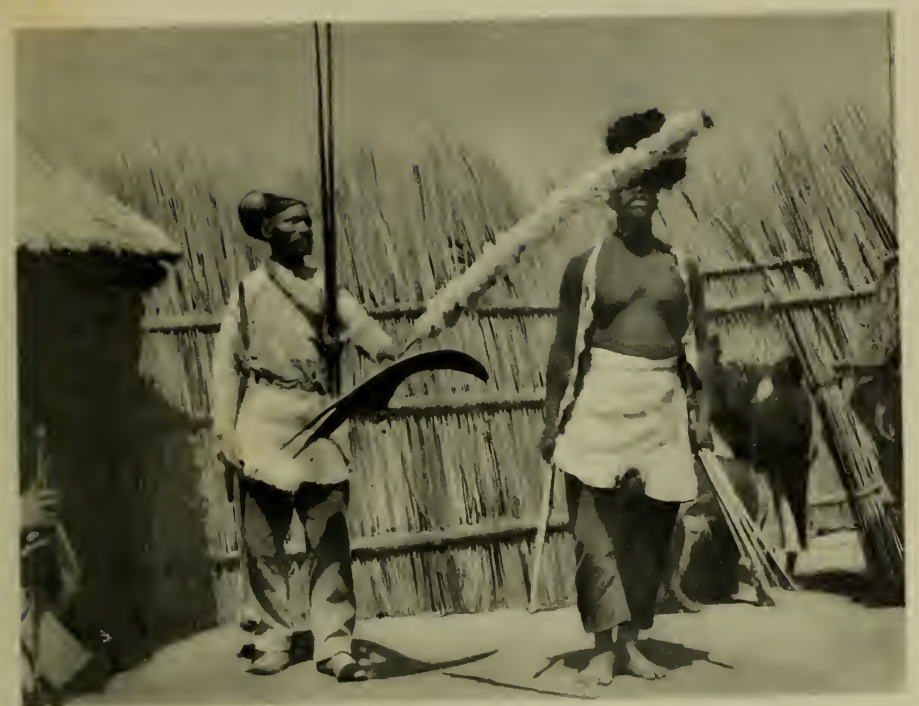

Une danse de guerre

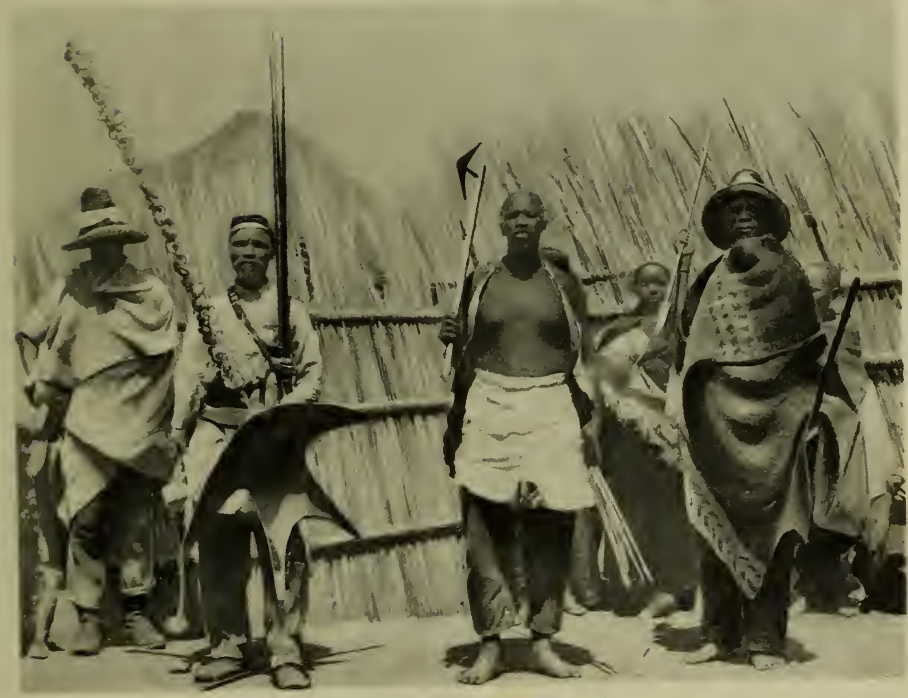

Les guerriers du chef Théko 


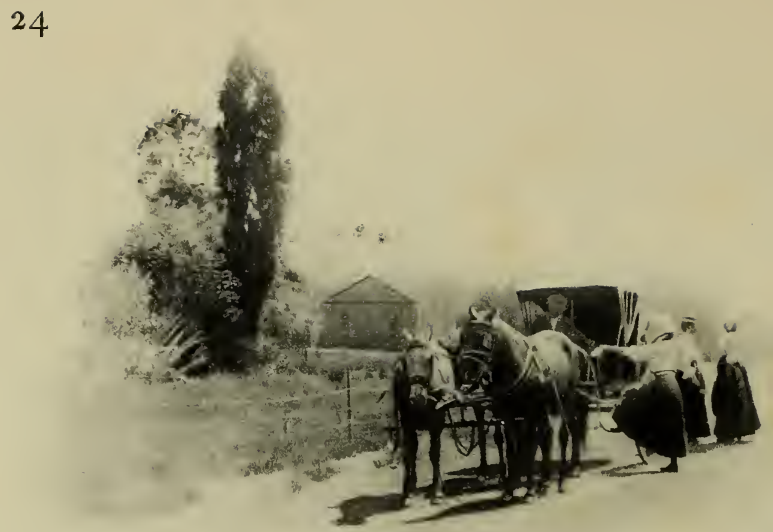

En route pour Maséru

station de $M$. et $\mathbf{M}^{\text {me }} \mathbf{F}$. Ellenberger dont les directeurs sont $\mathbf{M}$. et $\mathbf{M}^{\text {me }}$ Pascal, sans oublier l'annexe de la Pokané, à moitié chemin de la Sébapela, la station de montagne où se sont établis récemment M. et $\mathbf{M}^{\text {me }}$ Georges Dieterlen. Nous séjournons chez M. et $\mathbf{M}^{\text {me }}$ Christeller à la station de Béthesda et, par Maphéteng, nous effectuons notre retour à Morija.

Pendant les fêtes du Jubilé nous avions été les hôtes de M. Edouard Jacottet, puis de M. et $\mathrm{M}^{\mathrm{me}}$ R.-H. Dyke; actuellement nous jouissons de l'hospitalité de $\mathbf{M}^{\text {me }}$ Adolphe Mabille et de ses filles.

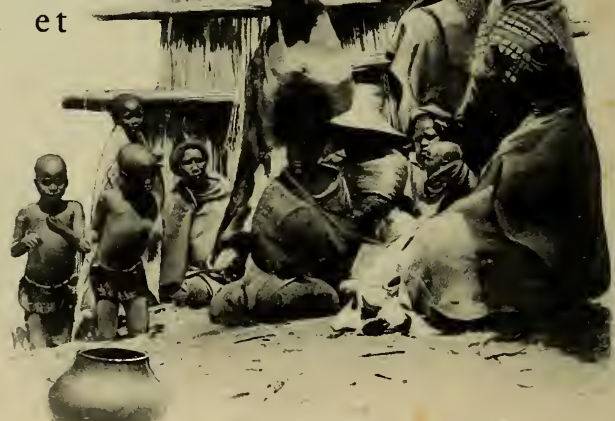

Une famille mo-Souto 
De Morija nous visitons à cheval la station d'Hermon qui possède la plus belle église du pays, l'œuvre de M. Christol; ce dernier, depuis son retour en Europe, a été remplacé par M. Bertschy.

$\mathrm{Au}$ cours de cette chevauchée nous avons rencontré des "Balé" jeunes filles païennes au corps recouvert de terre blanchâtre; ce sont des élèves d'une " école d'initiation ", cette institution païenne immorale qui perd heureusement du terrain grâce

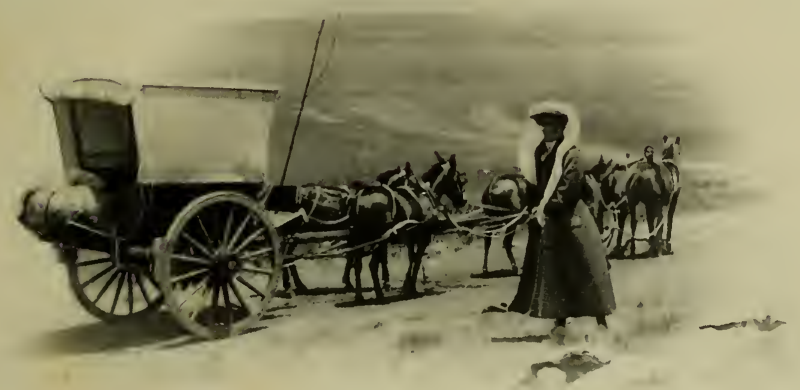

Par le "Post Cart", de Riverside à Matatiéle

à l'influence chrétienne. A l'annexe de Kolo nous trouvons le chef Mojela patriarcalement assis sous un arbre, entouré de ses subalternes avec lesquels il tient conseil; au moment du départ il me donne en présent sa hache de guerre.

Puis, nous séjournons encore chez $M$. et $M^{\text {me }}$ Baltzer, à la station de Thaba-Bosiou qui tire son nom de la montagne voisine, au sommet de laquelle le grand chef des ba-Souto, feu Moshesh, avait établi 


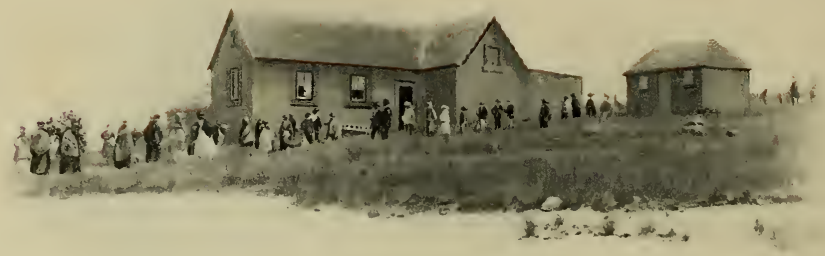

L'Eglise missionnaire de Mafubé

sa résidence; nous y voyons son tombeau. Nous n'oublierons pas non plus la visite au chef Théko qui, en notre honneur, donne l'ordre à deux de ses hommes de revêtir leurs costumes de guerre et de nous démontrer la manière dont ils se servent de leurs armes. Il me faudrait beaucoup de temps pour raconter en détail tout ce que nous avons expérimenté au cours de cet intéressant voyage missionnaire et décrire les belles assemblées auxquelles nous avons assisté, les nombreuses écoles que nous avons visitées; nous ne saurions assez remercier les missionnaires pour leur hospitalité et tout ce qu'ils ont fait pour nous. : Dois-je mentionner qu'en I 899, après un séjour avec M. Coillard, à Morija, j'avais vi- sité en sa compagnie la sta-

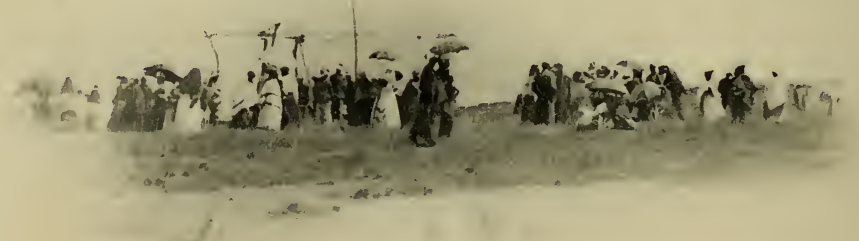

Culte de Noël à Mafubé 
tion de Cana qu'occupaient alors $M$. et $\mathbf{M}^{\text {me }}$ Kohler qu'ont remplacés $M$. et $\mathbf{M}^{\mathrm{me}}$ Jeanmairet. Puis, nous avions séjourné à Léribé où nous avions été les hôtes de $M$. et $M^{\text {me }}$ Hermann Dieterlen. Ainsi qu'on le sait, le fondateur de la Mission du Haut-Zambèze avait travaillé pendant vingt années à Léribé avant de se rendre sur les bords du grand fleuve.

Ensuite, afin de répondre à une invitation reçue, nous nous rendons dans l'East Griqualand. Pour effectuer ce parcours nous rejoignons la voie ferrée à Ladybrand, Etat d'Orange. Nous visitons en route

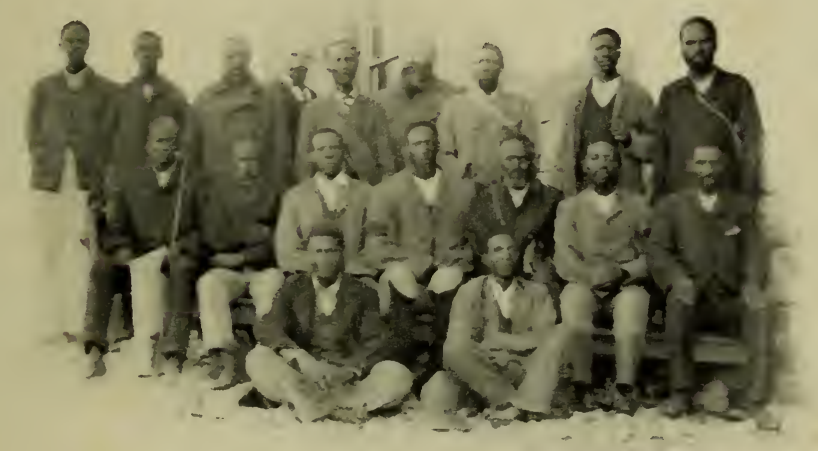

Assemblée du Consistoire de Mafubé

les champs de bataille de Ladysmith; par Pietermaritzbourg nous franchissons les hautes terrasses du Natal et nous atteignons Riverside, terminus de la voie ferrée. Nous entrons là dans l'East Griqualand, cette contrée des Griquas, considérée comme l'une 


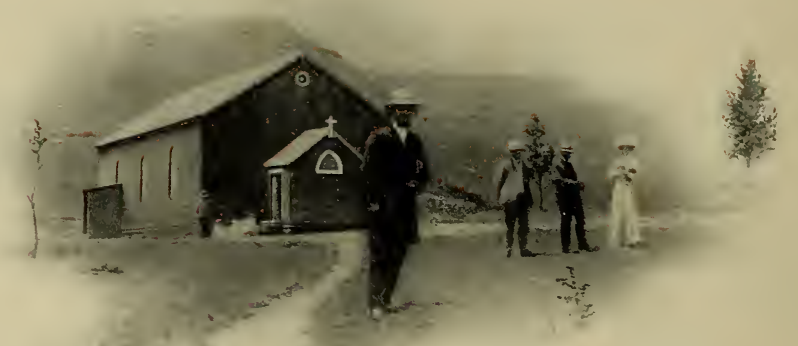

Station missionnaire

de Paballong

des meilleures au point de vue de la culture des c éréales et de l'élève du bétail.

De Riverside, par le moyen du "Post cart » attelé de six ou huit chevaux de relai, nous enlevons à grande allure, en une étape de douze heures, les cent trente-six kilomètres qui nous séparent de Matatiéle, une petite ville en formation où réside le magistrat anglais. De là, nous avons encore dix-neuf kilomètres que nous franchissons en deux heures pour atteindre Mafubé. Cette station missionnaire est située sur les contre-forts des Drackenberg, à la frontière est du Basoutoland. Nous y recevons un chaud accueil de ses directeurs, $M$. et $\mathbf{M}^{\text {me }}$ Paul Ramseyer, neuchâtelois.

Nous sommes arrivés à temps pour prendre part, à Mafubé, à la fête de Noël. Par une belle et chaude matinée d'été, cavaliers et piétons arrivent de toutes les directions, et, à l'heure du culte, l'église se trouve trop petite pour contenir les sept ou huit cents indigènes qui se groupent pittoresquement sur la prairie; 
les femmes sont revêtues d'étoffes aux couleurs voyantes et ont la tête recouverte de mouchoirs en forme de turbans bleus, verts, rouges et jaunes.

Les chevaux desselés paissent tranquillement dans les environs.

Comme au Basoutoland, nous constatons à Mafubé, la tranquillité, le sérieux de cette foule et l'attention prêtée à la parole du missionnaire. De Noël à Pâques nous avons fait, $M^{\mathrm{me}}$ Bertrand et moi, un séjour plein d'intérêt à Mafubé pour lequel nous ne saurions assez remercier $M$. et $M^{\text {me }}$ Paul Ramseyer. Grâce à eux, nous avons pu étudier dans ses détails, selon notre désir, cette œuvre si intéressante pour l'évangélisation des nombreux ba-Souto qui se sont réfugiés dans l'East Griqualand après les guerres civiles. La superficie de ce diocèse est presque équivalente à celle du canton de Neuchâtel; on y trouve quinze annexes avec sept cents enfants dans les écoles; nous les avons visitées

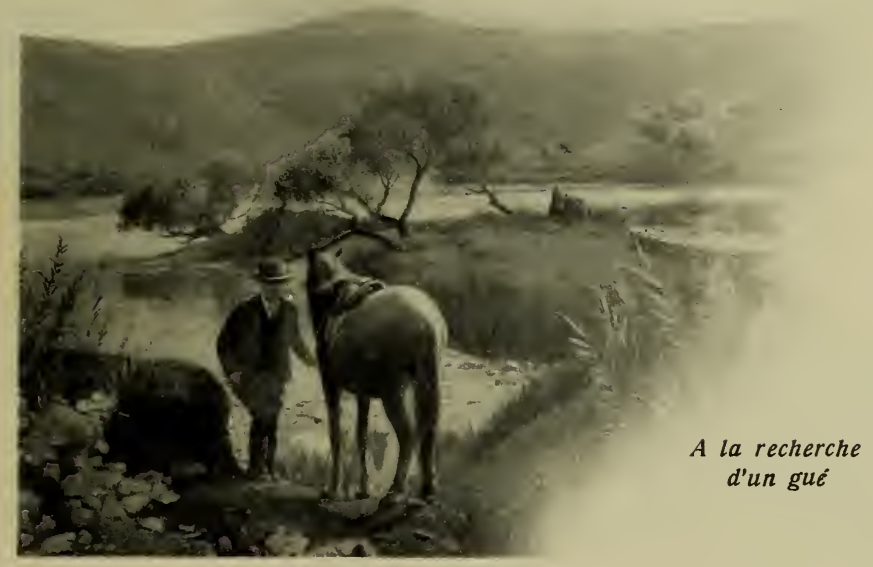


presque toutes, à cheval, avec M. Paul Ramseyer, et partout nous avons reçu le meilleur accueil des indigènes. - Notre dernière chevauchée a été à Mpharané, l'annexe la plus éloignée : environ quatre-vingts kilomètres aller et retour, en bonne partie à travers champs, y compris quatre rivières à passer à gué. Ces rivières ne sont pas toujours faciles à traverser surtout à l'époque du “Modupé» (pluies qui durent plusieurs jours), où très subitement l'étiage peut s'élever de plusieurs mètres; c'est ainsi que, près de Mafubé, un jeune cavalier mo-souto s'est noyé récemment.

N'avons-nous pas trouvé un jour le «facteur» qui devait porter nos lettres à la poste de Matatiéle, sur l'un des bords de la rivière, tandis que son cheval, qui avait perdu pied, avait gagné la rive opposée.

A Mafubé, j'ai assisté au consistoire formé par les évangélistes ainsi qu’à la réunion des instituteurs indigènes dont nous avons visité la plupart des écoles, et, comme au Basoutoland, j'ai eu le sentiment d'un travail sérieux et bien compris.

La tribu des ba-thépou restée jusqu'à maintenant très païenne commence aussi à ressentir l'influence

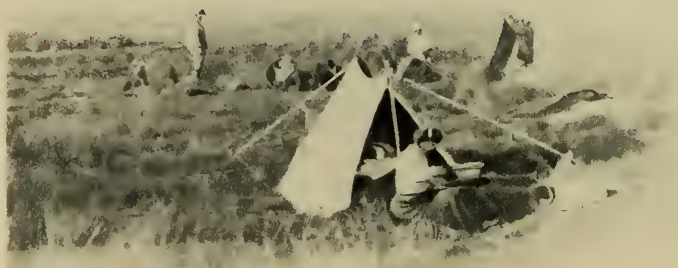




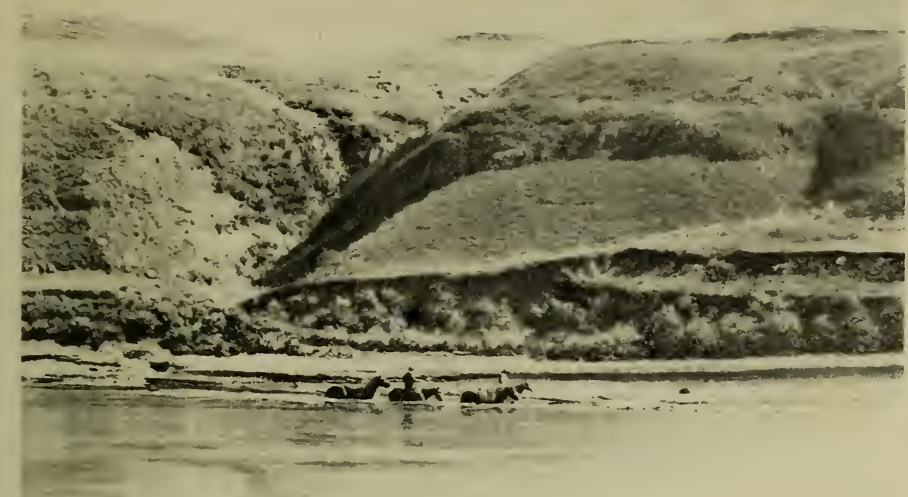

Traversée du Petit-Orange

bienfaisante qui se dégage de la station missionnaire de Mafubé. L'un de ces naturels ne nous disait-il pas en entendant une audition du phonographe : “... Il y a quelques années nous vous aurions tués comme étant des sorciers. "

De Mafubé, intéressante visite à la station de Paballoug que dirigent $M$. et $\mathbf{M}^{\text {me }}$ Moreillon et qui compte sept annexes et cinq cents écoliers.

Mafubé a été notre point de départ pour traverser à cheval le Basoutoland, de l'est à l'ouest, dans sa largeur, soit, de l'East Griqualand à l'Etat d'Orange.

Ainsi qu'on le sait le Basoutoland est bordé, à l'ouest et au nord, par l'Etat d'Orange; à l'est par le Natal et l'East Griqualand, et au sud par la colonie du Cap.

C'est un pays composé de montagnes tabulaires et de vallées profondes d'une âpre beauté.

On l'appelle à juste titre la Suisse de l'Afrique 
Méridionale; mais c'est une Suisse sans glaciers.

La superficie du Basoutoland est, à peu de chose près, égale à celle de la Suisse.

$\mathrm{Au}$ point de vue géographique, le Basoutoland offre un grand intérêt, car il est la clef de voûte de l'Afrique Australe et le centre de son système fluvial.

Deux grandes chaînes de montagnes traversent le Basoutoland du nord au sud. Ce sont:

a) Les Drackenberg qui, à l'est, forment sa frontière naturelle et qui constituent la ligne de partage des eaux de l'Océan indien et de l'Atlantique.

C'est dans les Drackenberg que se trouvent les montagnes les plus élevées de l'Afrique Australe, soit le Cathlin Peak (365o m.), le Giant castle (335o m.), le Mont aux Sources (335o m.). -- Sur le versant sud de cette dernière montagne, l'Orange et nombre de ses affluents de la rive droite, prennent leur source.

L'Orange, le plus important des fleuves de l'Afrique Australe, à un cours de mille six cents kilomètres.

A sa sortie du Basoutoland qu'il sillonne dans sa longueur, l'Orange suit la direction de l'ouest, pour draîner cette immense contrée d'une superficie de six cent mille kilomètres carrés, qui s'étend du Kalahari au nord aux montagnes de Nieweld au sud.

Avant de se jeter dans l'Océan Atlantique, l'Orange forme la frontière du Namaqualand, la colonie sudouest africaine allemande.

b) Les Malouti qui se ramifient en plusieurs chaînes parallèles. 


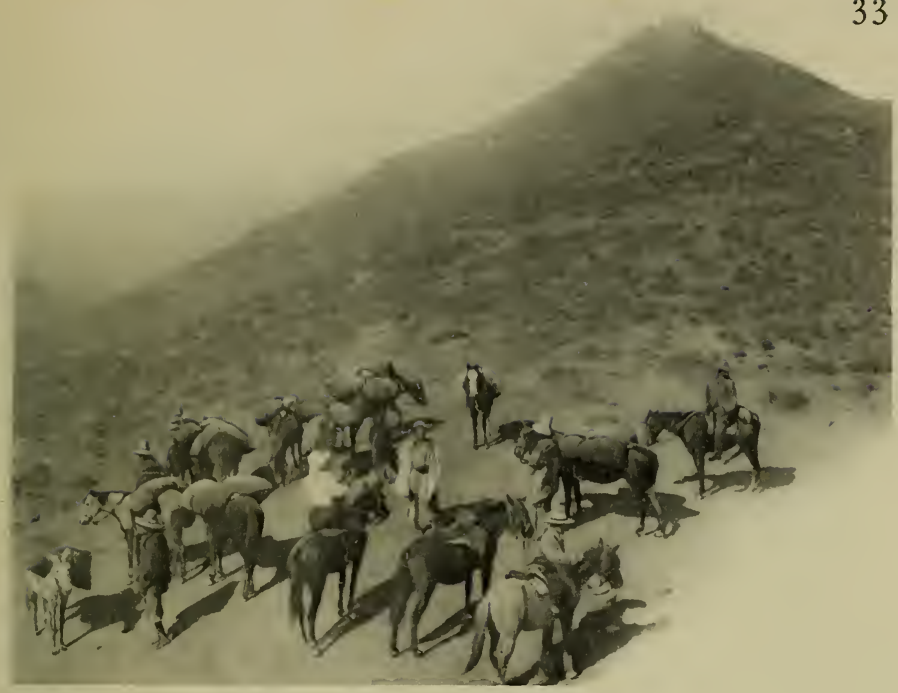

Nous rencontrons des indigènes

On peut sommairement diviser le Basoutoland en deux régions distinctes:

$\mathrm{I}^{\circ} \mathrm{La}$ région montagneuse proprement dite qui, des Drackenberg, à l'orient, est sillonnée par les chaînes parallèles des Malouti, entrecoupées de vallées où coule le fleuve Orange et ses affluents. Cette région, la plus sauvage, renferme de riches pâturages où paissent de nombreux troupeaux de chevaux, de bœufs et de vaches, de chèvres et de moutons.

$2^{\circ} \mathrm{La}$ région qui s'étend à l'ouest, entre les contre-forts des Malouti, et l'Etat d'Orange. Le HautPlateau, le Lessouto proprement dit, est une contrée fertile qui produit en abondance le blé, le maïs, l'avoine, le sorgho; elle est aussi la plus habitée.

Primitivement bergers, les ba-Souto ont franchi une étape supérieure et sont devenus agriculteurs. 
Le Haut-Plateau, ou le Lessouto, est certainement le pays africain qui, par rapport à sa superficie, occupe le premier rang au point de vue de la culture de la terre. Ce pays, non seulement nourrit ses propres habitants, mais il exporte chaque année au Transvaal et dans l'Etat d'Orange de cent cinquante à deux cent mille sacs de céréales.

D'une manière générale, le pays des ba-Souto est presque entièrement dénudé de bois et de forêts; cependant l'eau y abonde.

Ainsi que nous avons pu le constater nous-même à diverses reprises, les ba-Souto attribuent à l'œuvre chrétienne et morale qu'a exercée parmi eux la Société des Missions évangéliques de Paris, le fait qu'ils sont devenus un peuple.

Avant I 833 ce pays où régnaient encore le cannibalisme et toutes les horreurs du paganisme, était continuellement ravagé par les guerres civiles. C'est

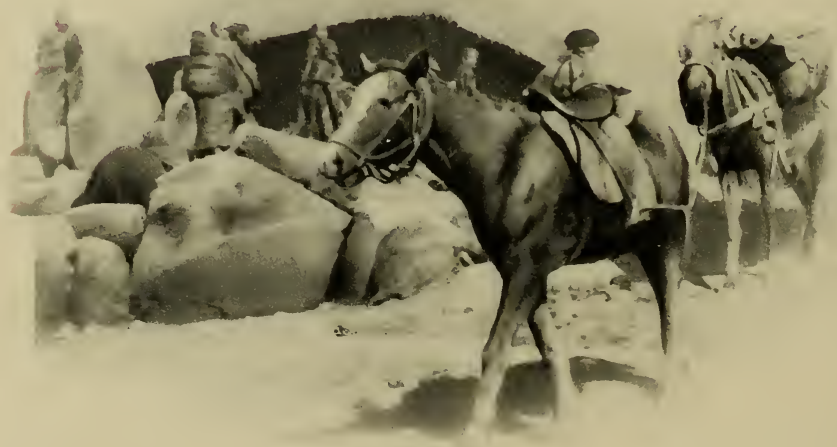

Nous arrivons chez le chef Raaleba 


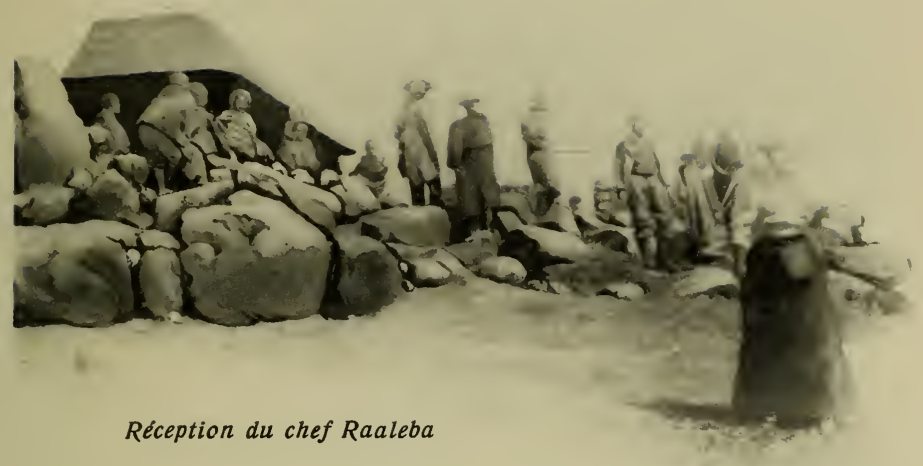

pendant la période qui s'écoula entre les années I 833 et I 870 que le grand chef Moshesh, fortement influencé par les préceptes du christianisme, rapprocha les différentes tribus décimées, soit les ba-Kuéna; les ba-Token: les ba-Sua; les ba-Taung; les ba-Puti; les ba-Rolong; les ba-Péli, etc.

De l'agglomération de ces clans, ou débris de clans, est née la nation des ba-Souto.

Le sé-souto, le langage des ba-Souto, l'une des nombreuses langues bantoues, est devenu, grâce aux travaux des missionnaires, une langue écrite qui aujourd'hui possède une littérature.

Pour en revenir à notre expédition, elle se composait de dix chevaux de selle dont quatre de bât qui transportaient les provisions, les tentes et autres objets de campement. Nous avions trois ba-Souto à notre service pour soigner les chevaux et l'un d'eux, "Kranye ", nous servait de cuisinier.

Notre ami, M. Paul Ramseyer, qui n'avait pas 


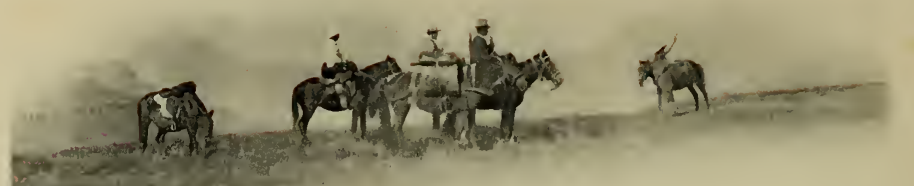

Nous dominons les gorges sauvages au fond desquelles serpente la rivière Malétsunyané

encore suivi cet itinéraire, nous accompagna; son concours nous fut précieux.

Nous partons le i 2 avril igog de Mafubé, et nous pénétrons par la frontière est dans le Basoutoland, au col de Quashas'neck qui fait déjà partie des Drackenberg. Nous acceptons ce soir-là l'hospitalité du magistrat anglais qui réside dans ce lieu d'une importance stratégique spéciale; je puis constater une fois de plus les bons services que me procure la lettre d'introduction collective provenant du ministère des colonies. Elle m'avait été aimablement remise lors de notre passage à Londres.

Le lendemain, après avoir chevauché à travers une série de monts et vallons, cultivés malgré l'altitude élevée, nous sommes en vue du fleuve Orange que nous traversons au gué de Nosi. A ce gué le fleuve forme une belle nappe d'eau transparente à la couleur d'émeraude d'environ cent cinquante mètres de largeur; le courant est fort et l'eau frange les flancs de nos chevaux.

Nous suivons la rive droite de l'Orange qui forme de gracieux méandres. Puis par un sentier abrupt, nous atteignons à nuit noire, l'annexe missionnaire 
de Tébellong qui est dirigée par un évangéliste indigène; il fait de son mieux pour bien nous recevoir et nous passons la nuit dans sa salle d'école.

Tébellong est un des plus excentriques avantpostes missionnaires de ces montagnes; il est situé dans un bel amphithéâtre bordé, au sud, par les Drackenberg et au nord par les Malouti. Au petit jour, alors que nos chevaux sont ramenés du pâturage, on nous présente quelques-uns des fidèles de Tébellong; puis nous montons en selle.

Après un col assez raide dont le faîte divise les territoires des chefs Makhaola et Griffith, nous entrons dans une contrée qui, avec la succession de ses montagnes et de ses vallées, donne l'illusion d'une mer orageuse qui se serait subitement solidifiée.

Nous rencontrons de nombreux ba-Souto à cheval,

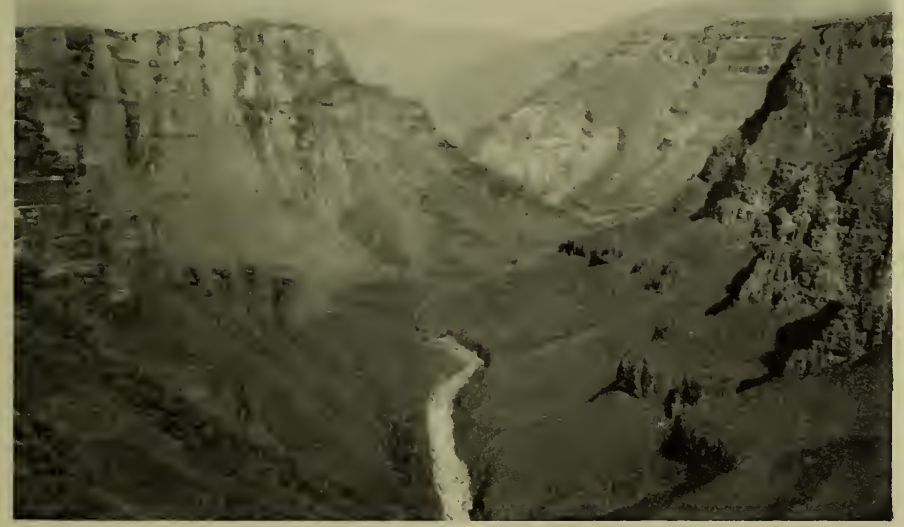

La vallée de la Malétsunyané 
drapés dans des couvertures de couleurs et coiffés de longs chapeaux en jonc ou paille tressés; ils escortent des chevaux de bât chargés de ballots de laine.

Au milieu de la journée, nous passons à gué le Qhabané, affluent de la rive droite de l'Orange et nous campons, dans la soirée, sur les bords du PetitOrange.

Le matin suivant, de bonne heure, notre caravane traverse le Petit-Orange ou "Senqunyané » ainsi que l'appellent les indigènes.

$\mathrm{Au}$ dernier village que nous rencontrons, voici une famille dont tous les membres nombre prodigieux d'enfants - sont couverts d'ocre des pieds à la tête.

Dans les pâturages aux teintes bronzées paissent de nombreux troupeaux de moutons et de chèvres angoras, fines et jolies, à la toison argentée.

Nous devons attaquer deux cols : le dernier surtout, celui de Thaba-Ntsu (Monta-

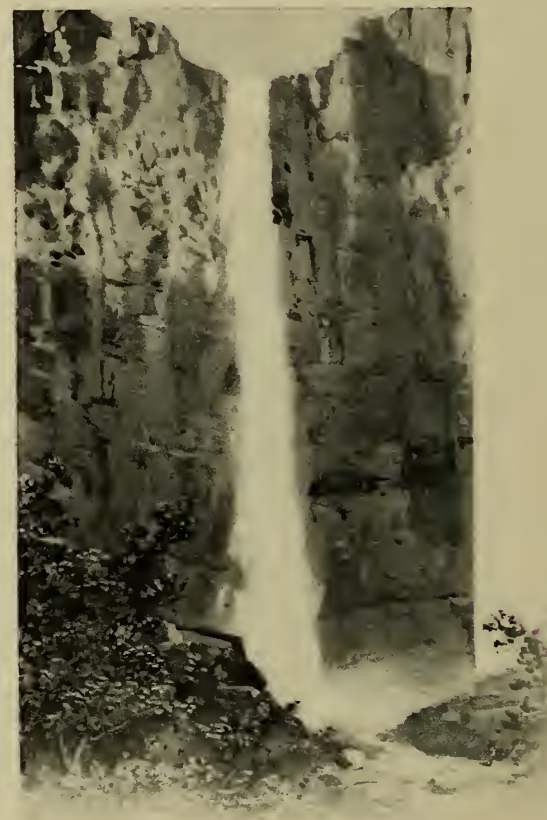

Chute de la Malétsunyané 


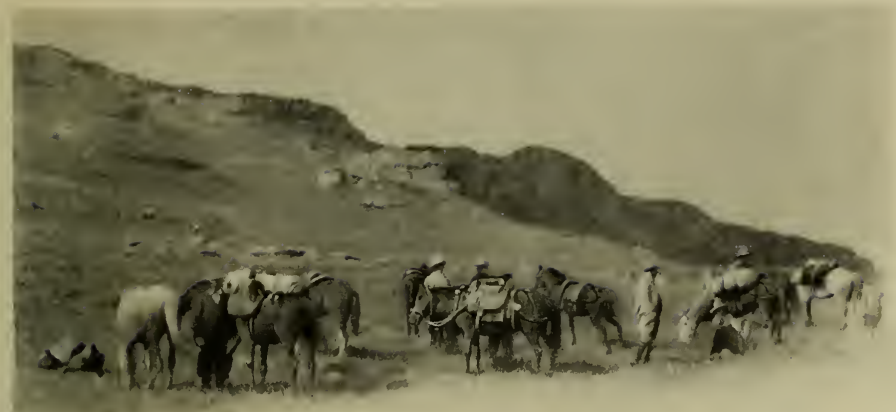

Notre campement près de la Chute

gne Noire) offre une longue grimpée raide, dangereuse, où nous admirons une fois de plus, l'agilité et la sûreté de pied de nos chevaux ba-Souto... un faux-pas pourrait avoir des suites fâcheuses!

Des aigles planent sur les rochers environnants.

Du faîte du col de Thaba-Ntsu, nous jouissons d'une vue étendue de l'est au sud sur la chaîne des Drackenberg. Devant nous, soit de l'ouest au nord, s'étendent les chaînes des Malouti.

I 6 avril. Froid la nuit dernière. Nos tentes militaires " tentes d'éclaireurs » aussi légères que possible, ne nous garantissent que bien imparfaitement. Ce matin, à cinq heures, un degré centigrade. Nous sommes de bonne heure en selle. Nous avons devant nous la vallée sauvage où coule la Malétsunyané, l'un des affluents de la rive droite de l'Orange.

Au milieu de la journée, nous atteignons le village du chef "Raaleba » (le père de la tourterelle). Il nous fait entrer dans sa hutte: sa hache de guerre, ainsi 
que son carquois rempli de lances, sont suspendus à la muraille à côté d'un mousqueton de cavalerie et d'un bandouilier vide de cartouches.

En qualité d'amis des missionnaires, "Raaleba» remplace trois de nos chevaux qui, trop fatigués, ne pourraient arriver au terme du voyage.

Chez le chef Raaleba comme ailleurs, les enfants, qui ne sont pas gênés par leurs vêtements, sont très nombreux.

Ici aussi les femmes portent sur la figure le

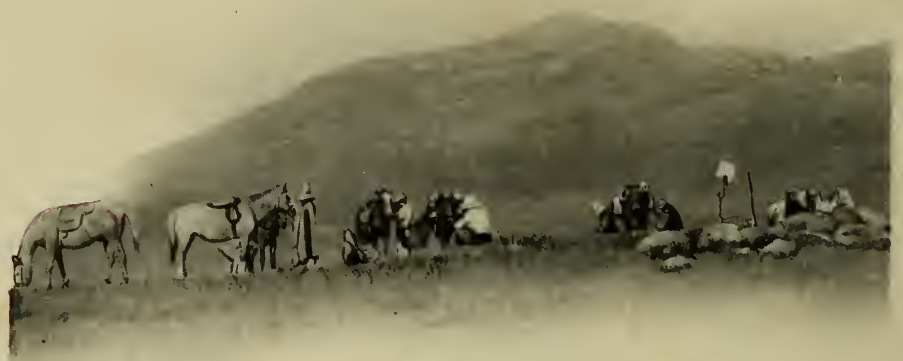

Le chef Raaleba nous amène des chevaux de rechange

tatouage en forme d'étrier: sur chaque joue trois raies noires qui partant de l'oreille, rejoignent soit le bas du menton, soit la bouche et la naissance du nez; une autre raie noire divise le front dans sa hauteur.

Nous ne tardons pas à surplomber les gorges sauvages, au fond desquelles serpente la rivière Malétsunyané après avoir franchi la paroi de rochers, qui forme, dit-on, les chutes les plus élevées du monde. 


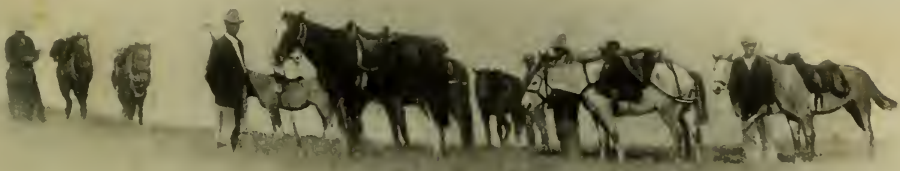

Le col de Thaba-Putsoa (Montagne grise)

Nous campons sur la rive droite de la Malétsunyané au sommet d'un plateau verdoyant.

Une fois les chevaux mis au pâturage et l'ordre établi au campement, nous descendons au milieu des éboulis et des pierres roulantes jusqu'au bas de la gorge d'où nous pouvons admirer la chute de la rivière Malétsunyané. Cet affluent de la rive droite de l'Orange rencontrant sur son passage une paroi verticale de rochers de cent quatre-vingts à deux cents mètres, se déverse en gerbes laiteuses dans l'enton-

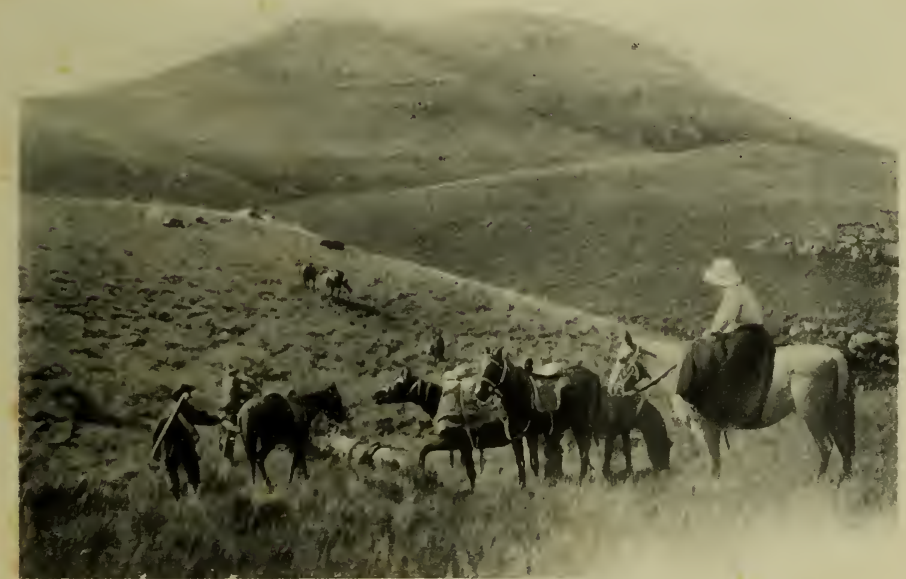

Un de nos chevaux enlizé dans les "Khafutso" ou marécages 
noir qui en forme la base et qui est encadré d'une luxuriante végétation.

I 7 avril. A six heures du matin, c'est-à-dire au moment où le soleil levant dorait les cimes qui nous entouraient, le thermomètre indiquait $+1 / 2$ degré centigrade.

Peu après le départ, les "Khafutso », marécages souvent traîtreusement recouverts d'herbes, nous arrêtent pendant quelques instants, et le cheval que je monte fait là une triste expérience : en quelques secondes il est enlizé jusqu'aux hanches.

Cette journée pourrait être appelée celle des "Cols"; nous avons dû en franchir quatre avant de gravir le plus important, celui de Thaba-Putsoa (Montagne Grise); il sépare la vallée de la Malétsunyané et celle qu'alimente un autre affluent de la rive droite du fleuve Orange, la rivière Makhaleng où nous allons pénétrer.

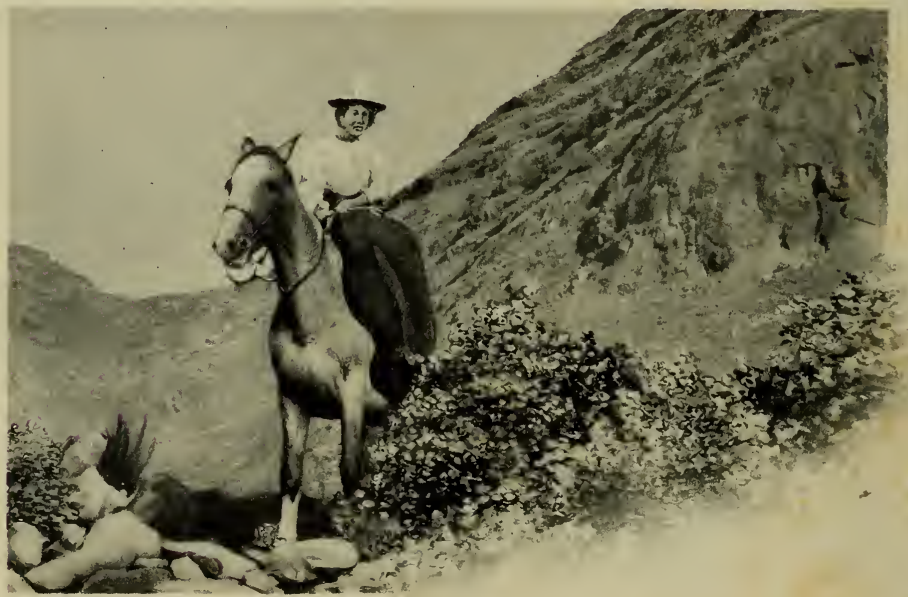

Après la chevauchée à travers les montagnes du Basutoland de l'Est à l'Ouest 


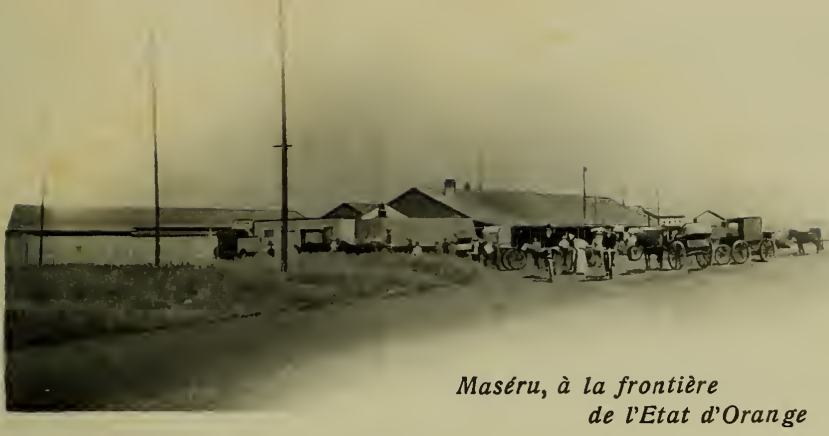

Devant nous s'étendent les montagnes du Lessouto proprement dit; elles sont pour la plupart coniques ou tabulaires. Comme à l'ordinaire les mauvais pas abondent. Dans les pâturages paissent de nombreux troupeaux de chevaux, de bœufs, de vaches, de chèvres et de moutons.

Le paysage ne manque pas de grandeur et nous rappelle les alpes vaudoises.

Nous campons au-dessus du village du chef Mpépé; plusieurs de ses sujets nous visitent et nous apportent du lait.

I 8 avril. Beaucoup moins froid; altitude moins élevée; à six heures du matin le thermomètre indique + I I degrés centigrade.

$\mathrm{Au}$ milieu de la journée nous atteignons le fond de la vallée où coule la rivière Makhalaneng, affluent de la rive droite de l'Orange. A mesure que nous avançons, les villages deviennent plus nombreux ainsi que les champs de blé, de maïs, de sorgho. En passant sur la rive droite de la rivière Makhalaneng, nous sommes sortis des montagnes Malouti. Ce soir 
nous campons au milieu du village du chef Cladi.

I 9 avril. Nous consacrons cette journée à franchir le grand plateau du Lessouto qui s'étend entre les contre-forts des Malouti et l'Etat d'Orange. A la nuit tombée, nous desselons nos chevaux à Maséru, la résidence du gouverneur anglais, à la frontière de l'Etat d'Orange, après avoir traversé le Basoutoland à cheval, de l'est à l'ouest.

20 avril. Le sous-gouverneur du Basoutoland m'a fait assiter aujourd'hui, à Maséru, à un “ Pitso ». C'est le "Conseil» des chefs ba-Souto qui étaient venus discuter la question actuelle et brûlante de "l'Union» c'es-à-dire de la Confédération des Etats de l'Afrique du Sud.

Ce n'était pas un spectacle banal que celui offert par ces cent trente grands chefs ba-Souto, délibérant d'une manière parfaitement digne et dans l'ordre le plus parfait.

A l'instigation des indigènes, des missionnaires et autres amis des ba-Souto, une pétition fut préparée pour que les droits des ba-Souto fussent reconnus et sauvegardés par la nouvelle Confédération des Etats du Sud-Africain.

Elle portait sur les points suivants :

I. Inaliénation du sol.

2. Prohibition de l'importation et de la vente de l'alcool.

3. Les revenus du pays des ba-Souto devront être affectés au bien des indigènes.

En I9o8, une députation composée de quatre 
grands chefs ba-Souto, accompagnés de leurs conseillers, ne craignit pas de franchir l'Océan et de se rendre à Londres pour présenter au Roi Edouard VII cette pétition, qui heureusement obtint gain de cause.

Nous avons rejoint la nouvelle ligne ferrée qui, de Maséru, relie Blœmfontein à Kimberley. Ces deux villes se sont beaucoup développées au cours de ces dernières années. Aujourd'hui, le "chariot de feu " comme les indigènes appellent la locomotive, a singulièrement rapproché le seuil de l'Afrique Centrale. C'est à Kimberley que nous avons pris place un mercredi soir, dans le "Zambesi Express", qui tous les mardis matin, après l'arrivée du steamer-poste d'Europe, part de la ville du Cap pour atteindre le samedi suivant, les chutes Victoria du Haut-Zambèze. C'est le premier tronçon du chemin de fer qui reliera un jour le Cap au Caire.

Le jeudi matin nous atteignons Maféking. Quels progrès! En 1895, l'expédition anglaise dont je faisais partie et qui devait explorer une partie du pays des ba-Rotsi, Haut-Zambèze, s'était orga- nisée à Maféking et

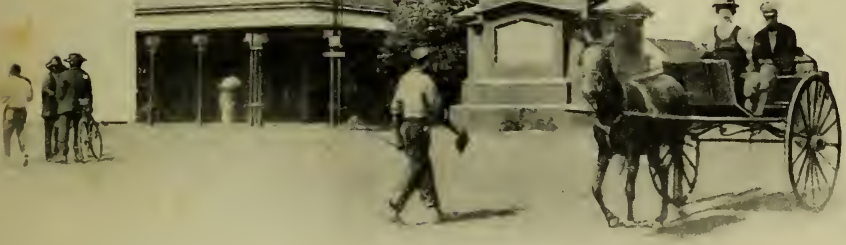

Monument commémoratif de la guerre des ma-Tébelé à Boulouwayo 
avait mis soixante-quatre jours avec des chariots traînés par des bœufs, ou à cheval, pour franchir les douze ou treize cents kilomètres qui nous séparaient du Haut-Zambèze, cela au milieu de difficultés sans cesse renouvelées.

Grâce à sa situation géographique, la petite ville de Maféling reste un centre commercial important. Nous entrons dans les pittoresques et verdoyantes collines de Lobatsi pour courir pendant toute la journée à travers les vastes plaines d'herbes jaunies, entrecoupées d'herbes et de buissons du Béchuanaland.

Dans la nuit nous passons près de Palapye, l'ancienne résidence de Khama, le sage roi des béChuana, qui, sous l'influence de son missionnaire M. Mac-Kenzie, a pu épargner le fléau de l'alcool à son peuple. Il est juste de dire qu'il a donné lui-même le bon exemple à ses sujets.

J'avais visité Khama, la première fois, en I 895 ; puis, plus tard, en I 899 , avec M. Coillard.

Nous franchissons la frontière du Matébéléland et, le vendredi, dans la matinée, nous nous trouvons en gare de Boulouwayo.

En I $89^{5}$, au retour

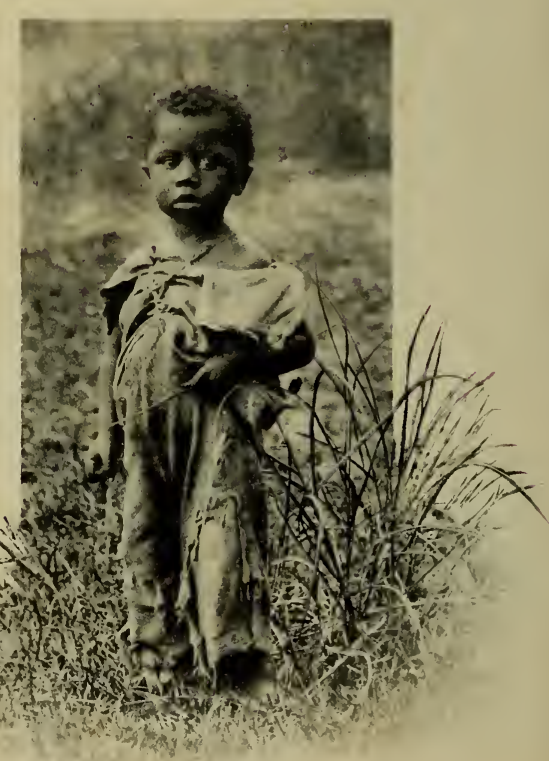

Un jeune ma-Tébéle 


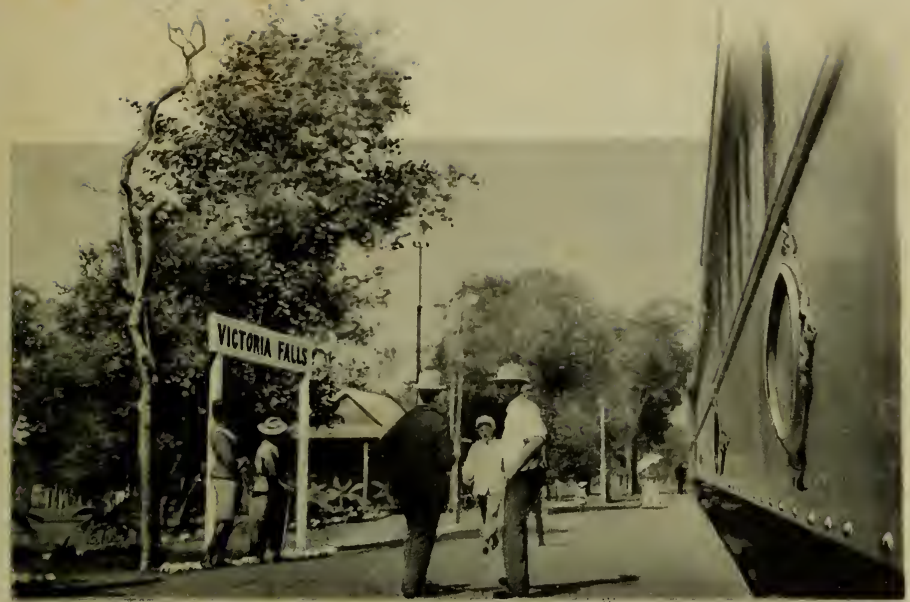

Station de la voie ferrée aux Chutes Victoria

de notre expédition au pays des ba-Rotsi, Boulouwayo qui fut notre premier point de contact avec la civilisation, était un "settle ment" de trois mois d'existence, où les huttes en tòle alternaient avec les tentes blanches et où l'on trouvait ici et là quelques constructions en briques rouges.

Aujourd'hui, à Boulouwayo, la propriété soumise à l'impôt représente une somme de cinquante millions de francs. Le "Mainstreet", rue principale, forme un large boulevard planté d'arbres et bordé de magasins où l'on trouve facilement tout ce qui est nécessaire à l'existence. Boulouwayo possède actuellement plusieurs églises, un hôtel-de-ville, une bibliothèque publique, un musée, etc.; il ne faut pas oublier un hôpital de premier ordre qui remplace avantageusement l'infirmerie primitive de laquelle pourtant plus d'un voyageur malade des fièvres a gardé un souvenir reconnaissant.

Nous nous rendons à la résidence qu'habite le 


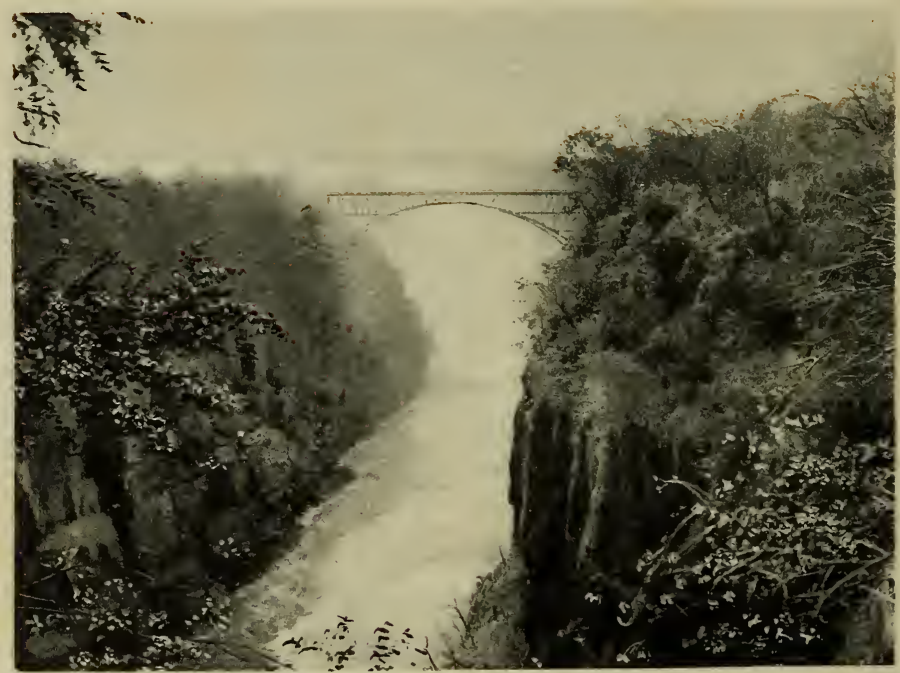

Sur le Haut-Zambeze, pont du chemin de fer de la ligne du Cap au Caire

premier magistrat. Elle est précédée d'une superbe avenue de conifères de plus de quatre kilomètres. Nous voyons dans le parc l'arbre à l'ombre duquel s'asseyait Lo-Bengula, le cruel roi des Ma-Tébélé, lorsque, entouré de ses chefs, il tenait ses conseils sanguinaires.

En I 893 " Boulouwayo ", qui signifie dans le langage du pays l'endroit où l'on tue, était encore le "Head Kraal», autrement dit la capitale de LoBengula.

Bien installés dans un confortable sleeping-car, nous franchissons à toute vapeur d'immenses plaines ondulées, fréquemment recouvertes d'herbes et de buissons qui cachent un sable blanc et épais, où jadis 
les attelages peinaient et souvent mouraient de soif. Nous nous souvenons de cette "Piste de la soif" longue de quatre-vingts à cent kilomètres, où, en I 895 , nos malheureux attelages, bœufs de trait et chevaux de selle, souffrirent de la soif pendant trois jours et trois nuits sans pouvoir être abreuvés. Pour sauver nos vies, nous dûmes abandonner notre grand chariot dans les sables, comme on abandonne un vaisseau sur la haute mer.

Samedi matin nous stoppons à la station du chemin de fer des chutes Victoria! Elle n'est pas banale cette station perdue dans la forêt : elle se compose d'une construction basse posée sur pilotis; à côté, et du même style, le bureau des postes et télégraphes. Non loin de l'emplacement où nous

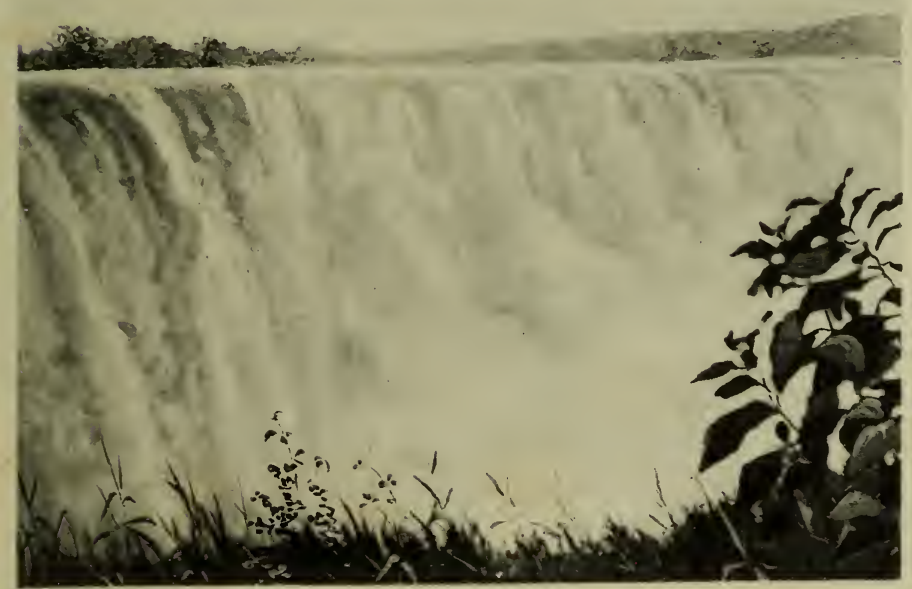

La Chute principale 
élevions jadis nos tentes, se dresse le «Victoria Falls Hôtel " qui comprend plusieurs " bungalows » à un seul rez-dechaussée, entouré de larges vérandahs ouvertes. Ces bungalows se dis s imulent dans la verdure et ne déparent en rien le paysage grandiose, digne cadre des chutes Victoria. De la terrasse

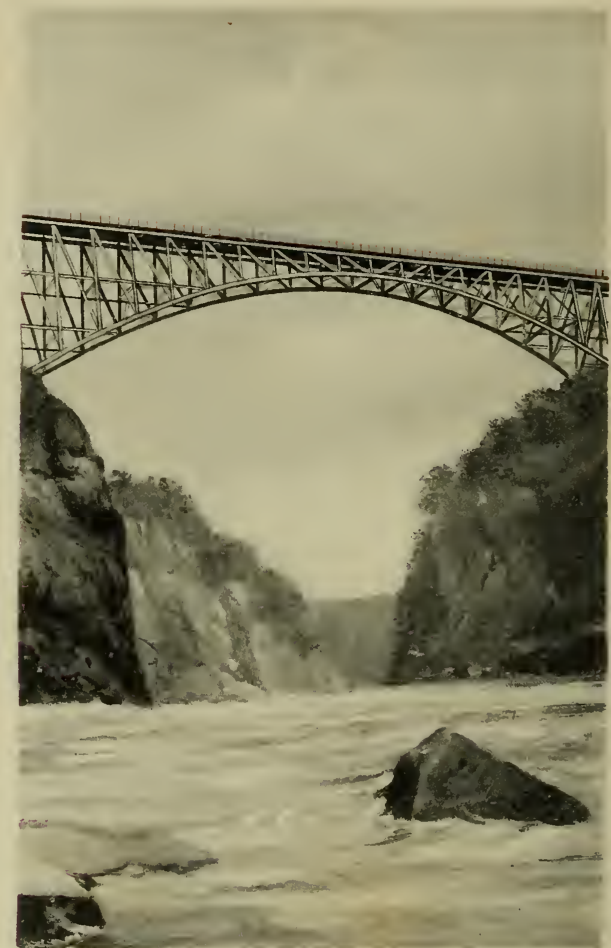

Le pont s'élève à $118 \mathrm{~m}$. au-dessus du fleuve. centrale, nous avons sous les yeux la forêt qui descend en pente douce jusqu'au Zambèze. A notre gauche nous apercevons les immenses colonnes de vapeur opaque qui se dégagent des chutes dont nous entendons le sourd mugissement.

Nous voyons devant nous le nouveau pont du chemin de fer, le plus élevé du monde à ce qu'on dit. Il s'élève à environ cent dix mètres au-dessus du fleuve et se compose d'une seule arche que l'on 
comparait récemment à un mouchoir de dentelle jeté d'une berge à l'autre.

Le pont traverse le fleuve en aval des chutes qu'il ne dépare en aucune manière, et il peut à juste titre être considéré comme une merveille de l'industrie. Il a été inauguré en 1905, soit cinquante années après que Livingstone eut découvert les chutes auxquelles il donna son nom. Les indigènes, dans leur langage imagé, les appellent "Mousi-oa-Thounya», la fumée qui tonne.

Nous avons employé une semaine à les étudier et à les admirer sous toutes leurs faces. Dans cette saison, en mai, après la saison des pluies, le Zambèze roule une masse d'eau encore bien plus considérable que lorsque, pour la première fois, en octobre 1895 ,

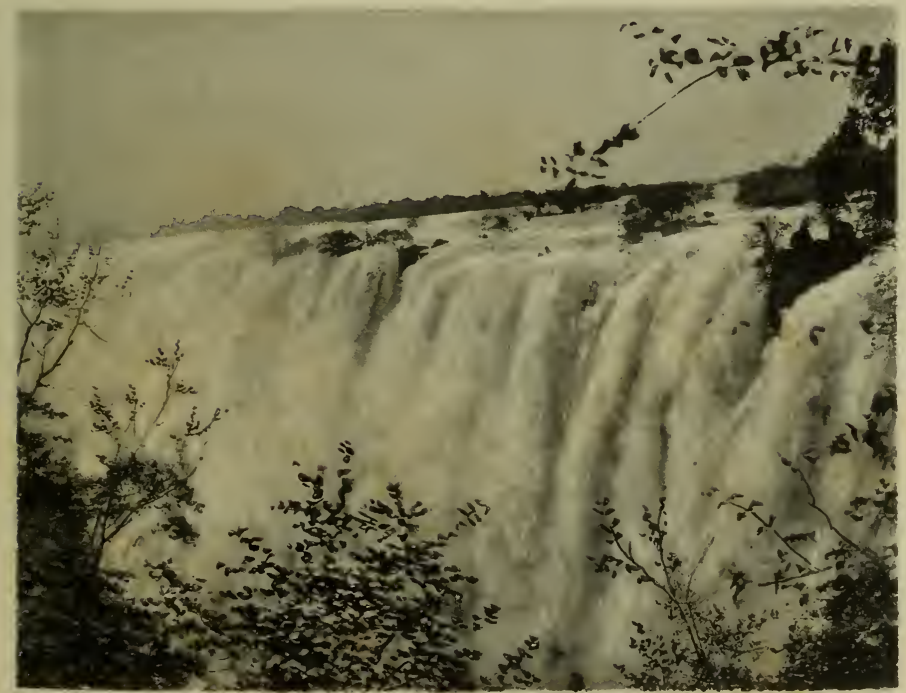

La Chute de l'arc-en-ciel 
soit en pleine saison sèche, je vis les chutes Victoria.

On doit se rappeler qu'en amont des chutes, le fleuve très large rencontre sur son passage une falaise ou plutôt une gigantesque fissure creusée dans la roche basaltique qui s'étend sur un profil de mille six cents mètres et qui a une hauteur de cent à cent vingt mètres.

Cette énorme masse d'eau écumante, projetée dans l'abîme, rebondit contre la paroi opposée du gouffre, et tourne à angle droit pour atteindre l'issue qu'elle s'est creusée, à l'est, entre deux parois de rochers, à l'endroit appelé "Boiling pot ".

Pour se faire une idée d'ensemble des chutes et les voir de face, il faut suivre la crête sud du gouffre, la "Rain Forest), couverte d'une forêt tropicale où les myrtes, les palmiers-phœnix, les nombreuses espèces de fougères, les orchidées, etc., croissent au pied de grands figuiers.

Si nous partons de l'ouest, nous voyons la première cataracte "Leaping water ", que deux petites îles verdoyantes séparent de la chute principale, le «Main Fall »; l'île Livingstone se trouve entre cette dernière et le "Rainbow ", soit la chute de l'arc en ciel.

Les indigènes appellent cetțe forêt l'endroit où la pluie est née; en effet, quoique le ciel soit d'un bleu d'azur, nous sommes entourés de nuages opaques qui retombent sur nous comme une pluie d'été. Lorsque, ici et là, ces nuées se déchirent on a de magnifiques aperçus sur cette masse d'eau ambrée ou blanche comme neige, qui, avec grand fracas, se 


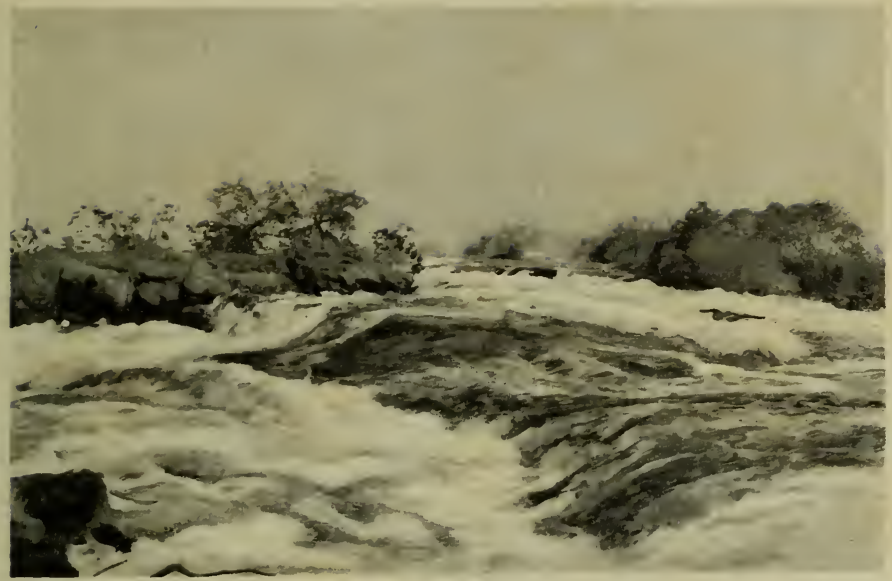

Le Zambeze en amont des Chutes Victoria

déverse en gerbes gigantesques dans l'abîme que nous avons à nos pieds; le soleil s'y jouant, produit des effets de lumière merveilleux.

En sortant, à l'est de la "Rain Forest", on domine le gouffre où les eaux en furie convergent de toute la ligne des chutes et s'amoncellent bouillonnantes et mugissantes. De là le Zambèze se précipite en formant de nombreux méandres dans la gorge sauvage de Bakota pour reprendre peu à peu sa course vers l'Océan Indien.

Parfois les colonnes de vapeur s'élèvent à plus de trois cents mètres et on peut les apercevoir jusqu'à quatre-vingts kilomètres de distance.

$\mathrm{La}$ végétation qui entoure les chutes est d'une grande beauté.

La faune n'est pas non plus à dédaigner. Un 
crocodile a été tué il y a quelques jours sur le pont même du chemin de fer.

On a calculé que la force engendrée par les chutes représente trente-cinq millions de chevaux. Les chutes Victoria pourront devenir, dans cette partie de l'Afri-

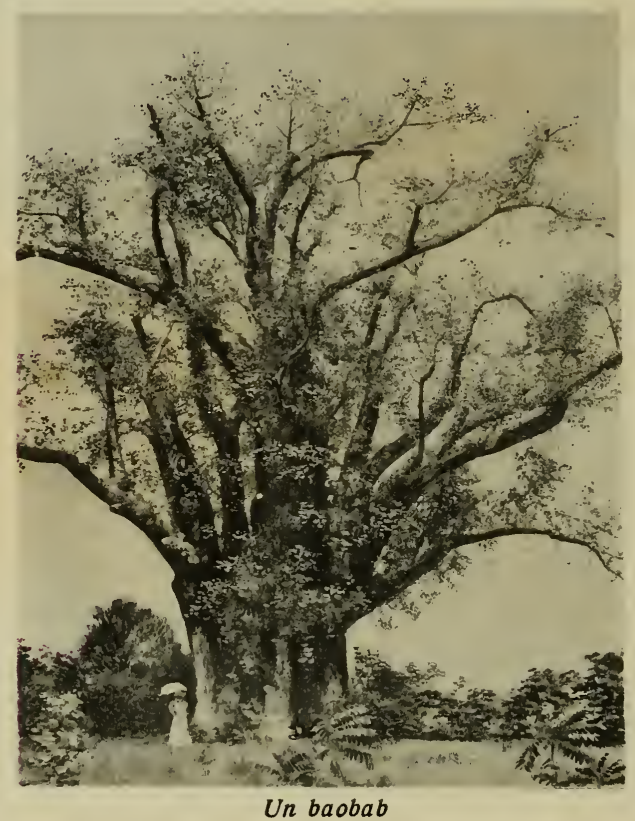

que, un puissant réservoir de force électrique. Déjà une société constituée, la "Victoria Falls Power Company ", projette d'y établir prochainement une installation électrique pour la distribution d'électricité dans les contrées environnantes. Hâtons-nous d'ajouter que ce projet a été conçu de manière à ne nuire en rien aux beautés naturelles des chutes du Haut-Zambèze. 
Cette compagnie étudie aussi les moyens de transmettre un jour l'électricité jusqu'au "Witwatersrand " le district des mines du Transvaal.

Actuellement la voie ferrée atteint la frontière sud-est du Congo belge à l'endroit appelé "Star of the Congo " et selon toutes probabilités elle rejoindra rapidement le lac Tanganyka pour traverser l'Afrique dans toute sa longueur reliant ainsi le cap au Caire, l'une des plus audacieuses entreprises que l'esprit humain ait jamais osé concevoir. Pour ce qui concerne le développement et l'exploitation rationnelle de ces vastes contrées, hier encore perdues dans le centre africain, le rôle des réseaux de voies ferrées peut être comparé à celui que jouent dans le corps humain les veines et les artères.

Ainsi qu'un éclaireur diligent, le fil télégraphique, "African Transcontinental Telegraph ", devance la ligne du chemin de fer, et, d'Europe, il est possible aujourd'hui de lancer un télégramme jusqu'à Ujiji, situé à l'est du lac Tanganyka, la localité où Stanley rencontra Livingstone.

Par un coucher de soleil où les eaux du Zambèze devenaient pourpres, puis brillantes comme de l'or en fusion, nous avons franchi en canot les dix kilomètres qui, en amont des chutes, nous séparaient de la station missionnaire de Livingstone où nous avons fait un séjour plein d'intérêt chez $M$. et $M^{\text {me }}$ Louis Jalla. Ainsi qu'on le sait, M. Louis Jalla, et son frère cadet, $M$. Adolphe Jalla, ont été parmi les premiers collaborateurs du regretté M. Coillard, 


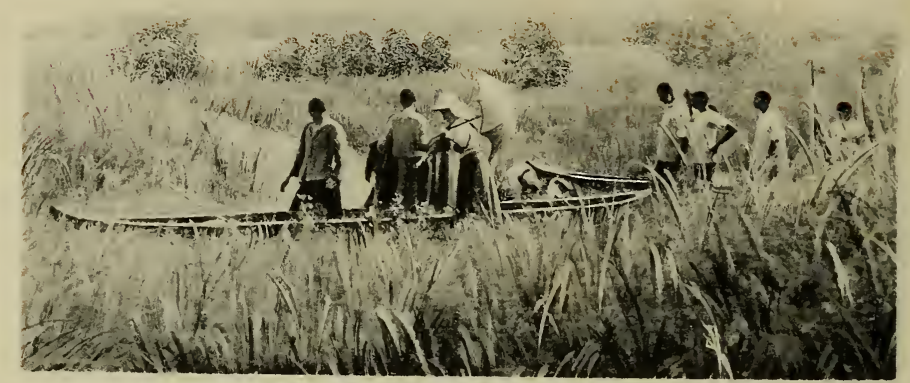

Nous débarquons près de la station missionnaire de Livingstone

le fondateur de la Mission de pionniers du HautZambèze. Quoique jeune encore, M. Louis Jalla est aujourd'hui, non seulement le vétéran des missionnaires du pays des ba-Rotsi, mais encore le doyen des blancs qui habitent cette région.

Outre son œuvre sur la station même, M. Louis Jalla a aussi entrepris un travail intéressant parmi les jeunes noirs qui habitent le "settlement" de Livingstone. C'est la tête de ligne de la voie terrée du cap au Caire. Livingstone, cette ville née d'hier, dont les premiers fondements ont été creusés en 1905, aujourd'hui résidence du gouverneur, est déjà éclairée à l'électricité. Elle sera prochainement dotée d'une grande église. Elle possède une gare, un hôpital, un bureau des postes et télégraphes et plusieurs magasins.

Les autorités reconnaissent que les crimes et les délits ont diminué dans une notable proportion depuis que M. Louis Jalla a créé une école du soir 
au "settlement» de Livingstone. Cette école, ainsi que les cultes, ont lieu dans le "Coillard's Memorial Hall ", jolie chapelle démontable que les amis anglosaxons de M. Coillard lui avaient envoyée d'Eu rope. Bonne manière, il nous semble, de perpétue r la mémoire du missionnaire-pionnier du Haut-Zambèze.

Tout récemment, et près d'ici, quatre voyageurs qui remontaient le Zambèze en canot ont fait naufrage par suite de l'attaque imprévue d'un hippopotame. Léwanika, le roi des ba-Rotsi, qui réside à la

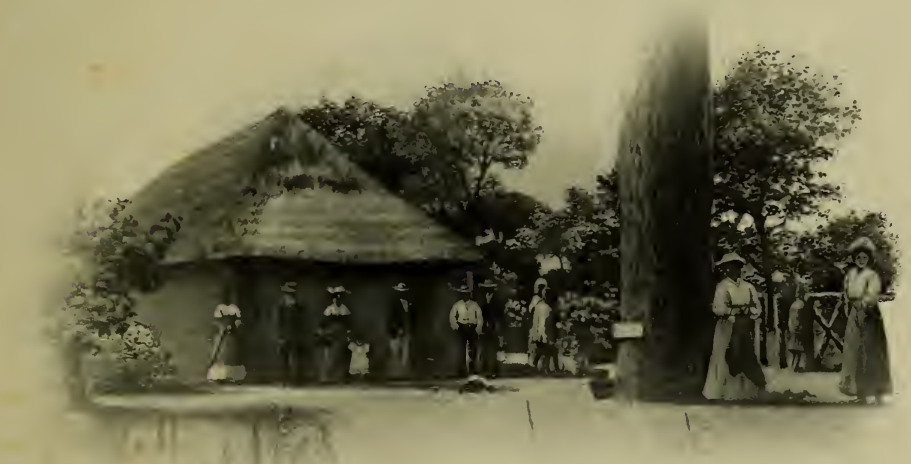

A la station missionnaire de Livingstone

capitale Léalouyi, située de quatre à cinq cents kilomètres au nord-ouest des chutes Victoria, se considérait, il y a peu de temps, comme le souverain absolu d'une vingtaine de tribus dont il avait fait ses vassales par la force des armes.

$\mathrm{Au}$ point de vue géographique, la superficie de son royaume était limitée au nord par la ligne du partage des eaux du Congo et du Zambèze; à l'est 
elle confinait à la rivière Kafoukué, et, à l'ouest au vingtième degré de longitude est de Greenwich; sa frontière sud était formée naturellement en partie par le Zambèze et le cours du Linyanti, ou Chobé. Par conséquent le pays des ba-Rotsi était approximativement compris entre le douzième et le dixhuitième degré de latitude sud et le vingtième et le vingt-neuvième degré de longitude est de Greenwich.

En r 905 , au cours d'un différend survenu entre le Portugal et Léwanika à propos de la frontière est de la colonie d'Angola et de la frontière ouest du pays des ba-Rotsi, le roi d'Italie, qui avait accepté cet arbitrage, a réduit la frontière ouest du pays des ba-Rotsi jusque près du vingt-deuxième degré est de Greenwich.

Aujourd'hui, d'après les traités que le roi Léwanika a conclus avec la "British South Africa Company ", son royaume est compris dans la Rhodésia du nord-ouest.

En 1895 , le roi Léwanika était un autocrate absolu qui avait droit de vie et de mort sur chacun de ses sujets. Les habitants, le sol et ce qu'il renferme, y compris les produits de la terre, tout appartenait théoriquement au roi. Aujourd'hui le royaume des ba-Rotsi est divisé en districts, à la tête de chacun desquels se trouve un officier civil; leur supérieur hiérarchique, l'administrateur, réside au siège du gouvernement, à Livingstone, la nouvelle ville fondée sur la rive gauche du Zambèze, non loin des chutes Victoria. 


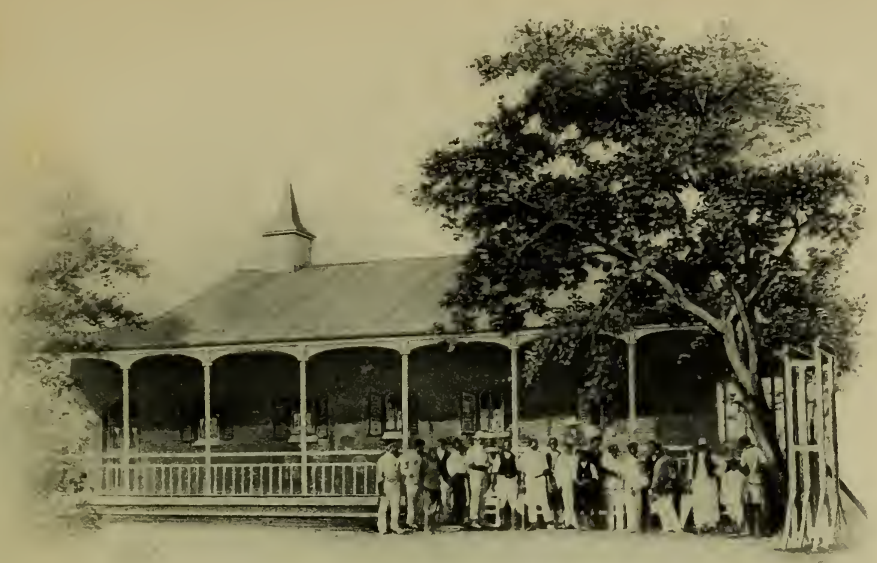

La chapelle érigée au "settlement " de Livingstone en souvenir de M. Coillard

L'administrateur exerce un contrôle et une surveillance sur tous les chefs de district et les questions importantes lui sont soumises. Il compte parmi ses principaux collaborateurs l'officier d'état civil, le commandant de la police indigène, le médecin, le directeur des postes et télégraphes et celui des douanes. Aujourd'hui le royaume des ba-Rotsi, autrement dit la Rhodésia du nord-ouest, bénéincie du régime des douanes.

Pour le moment, Léwanika et ses chefs exercent encore la juridication civile, mais il est probable qu'elle passera peu à peu entre les mains des officiers civils européens.

D'après la convention conclue avec la "British South Africa Company ", la Rhodésia du nord-ouest a été ouverte à la colonisation, sauf la vallée du bo-Rotsi proprement dite où se trouve la capitale Léalouyi et où réside Léwanika. Suivant la proclamation de mars 1904, les indigènes doivent payer une taxe annuelle de Fr. 12.50 et le produit des taxes est dépensé en grande partie, pour 


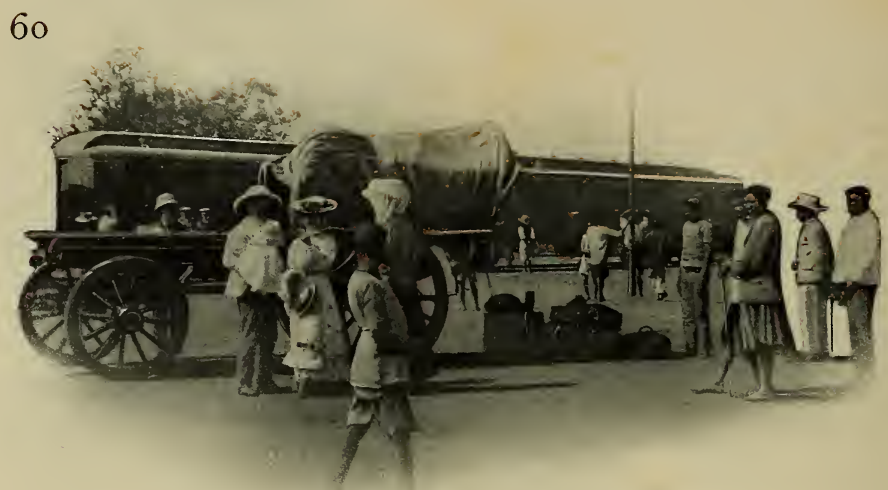

La gare $d u$ "settlement " de Livingstone

des services ou travaux publics dont profite le pays.

Prenons comme exemple ce qu'était le service des postes il y a douze années, et ce qu'il est aujourd'hui. Jadis, au pays des ba-Rotsi, les lettres ne parvenaient à destination qu'après de longs mois et des risques sans fin: des indigènes, dressés à cet effet, et marchant deux par deux, les transportaient piquées au bout de longs bâtons. Il arrivait que pour échapper aux bêtes fauves ou autres dangers, ces facteurs d'un nouveau genre retournaient sur leurs pas et même parfois renonçaient à leur course.

Le service des postes et télégraphes, qui s'est récemment beaucoup développé dans la Rhodesia du nord-ouest, compte actuellement onze bureaux de poste proprement dits et huit bureaux d'où l'on peut télégraphier; en outre, des routes postales ont été établies dans les parties les plus éloignées du pays.

Au cours de l'exercice igo8-ıgog, les bureaux de poste de la Rhodésia du nord-ouest dépêchèrent I 63,766 lettres et en reçurent i 28,414. Le total des 


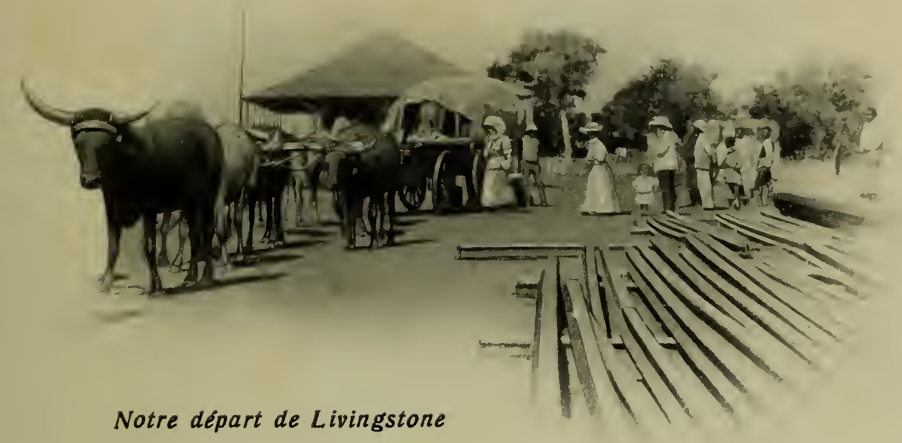

envois postaux de tous genres qui passèrent par ces bureaux se monta pour les expéditions à 187,772 , et le nombre des réceptions atteignit le chiffre de 199,433 .

C'est en 1895 , au cours du voyage d'exploration fait dans cette partie de l'Afrique, que, à Léalouyi, capitale du pays des ba-Rotsi, j'eus le privilège d'être pendant plusieurs semaines, dans sa hutte, l'hôte de feu le missionnaire-pionnier Coillard, l'émule de Livingstone.

M. Coillard m'avait mis en relation directe avec le roi Léwanika.

Il faut rendre justice à l'œuvre de civilisation chrétienne que, sous les auspices de la Société des Missions évangéliques de Paris, $M$. Coillard et ses dévoués collaborateurs ont entreprise dans ces contrées sauvages, où surtout pendant les premières années, ils eurent à lutter contre des difficultés et des souffrances sans cesse renouvelées dont nous n'avons aucune idée en nos pays civilisés. 
Aujourd'hui, quoique n'étant pas encore chrétien, Léwanika à été pourtant transformé par le christianisme, lui qui, il y a peu d'années, était un despote cruel et sanguinaire. Il avait un code de supplices plus horribles les uns que les autres; toutes ces horreurs sont choses du passé.

Jadis Léwanika célébrait le renouvellement de la lune en se livrant avec ses chefs à de terribles scènes d'orgies, souvent terminées par le meurtre. Or, depuis quatorze ans, il n’a pas touché aux boissons spiritueuses.

Léwanika tirait autrefois le plus clair de ses revenus de razzias d'esclaves, coutume barbare à laquelle il a renoncé depuis longtemps. De plus, il a libéré, en 1906, les esclaves ou serfs domestiques qui se trouvaient dans le pays.

Pour nous qui jouissons des bénéfices de dix-neuf siècles de christianisme, il est difficile de nous représenter tout ce que le paganisme entraîne d'horreurs à sa suite. On peut affirmer, sans se tromper, que la définition du paganisme est l'égoïsme poussé juqu'au cynisme le plus absolu.

Quoi qu'on en dise, le principe de la charité est un produit direct du christianisme et il ne se rencontre à aucun degré en contrée païenne.

Par charité, nous entendons surtout parler des rapports qui régissent les êtres humains entre eux, et non pas des hôpitaux, des établissements hospitaliers de tous genres qui abondent dans nos pays civilisés. En pays païens on trouve, autant d'orphelins, de 


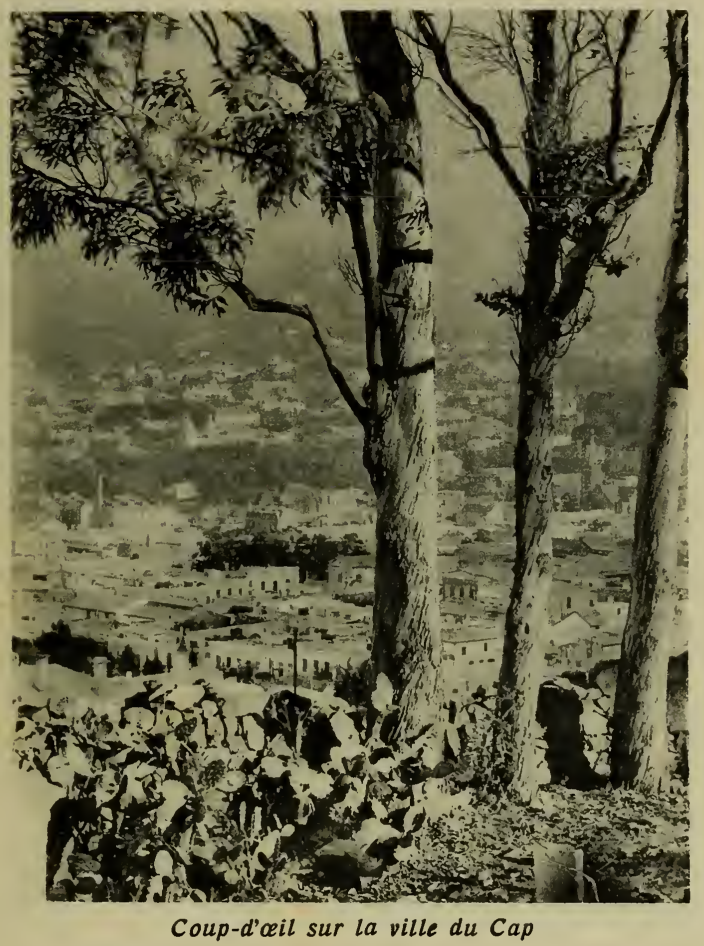

miséreux, d'estropiés et d'aveugles qu'en Furope ou aux Etats-Unis; mais personne, hors les missionnaires, ne se préoccupe du sort de tous ces malheureux. On ne trouve les vrais principes de la charité pas plus chez les ba-Rotsi païens du HautZambèze que chez les sectacteurs de Confucius, en Chine, ou chez les boudhistes aux Indes.

En assistant à la célébration du Jubilé de Morija et en parcourant le pays, tout voyageur aurait pu constater les résultats encourageants que la Société 
64

des Missions évangéliques de Paris peut enregistrer au pays des ba-Souto après soixante-quinze années de persévérant labeur; cette œuvre missionnaire a engendré celle qui a été entreprise sur le Haut-Zambèze au pays des ba-Rotsi.

Il y a vingt-cinq années à peine que les missionnaires de la Société des Missions évangéliques de Paris ont apporté les principes du christianisme dans cette région du Haut-Zambèze.

Avant leur arrivée, le pays des ba-Rotsi pouvait être appelé un pays de sang; aujourd'hui plus de trente missionnaires et aides-missionnaires habitent huit stations échelonnées le long du Zambèze sur un parcours de cinq cents kilomètres.

Ces stations peuvent devenir des centres d'évangélisation, d'éducation, de transformation dans la manière de vivre et de penser, bref, des noyaux de civilisation chrétienne.

Quoiqu'il y reste encore beaucoup à faire et que les conversions proprement dites n'y soient pas nombreuses, n'est-ce rien que l'abolition de l'esclavage, la suppression de l'infanticide, du pouvoir des sorciers et des cruels supplices dont la pensée seule fait frémir d'horreur? Il faut arriver à la conclusion que dans nos pays civilisés, nous devons tout au christianisme qui, siècle après siècle, a profondément modifié notre manière de voir et d'agir.

Le $\mathrm{XX}^{\mathrm{me}}$ siècle sera, mieux encore que le $\mathbf{X I X}^{\mathrm{me}}$, le siècle où les Missions en pays païens prendront une grande envergure. Nous en avons eu récem- 


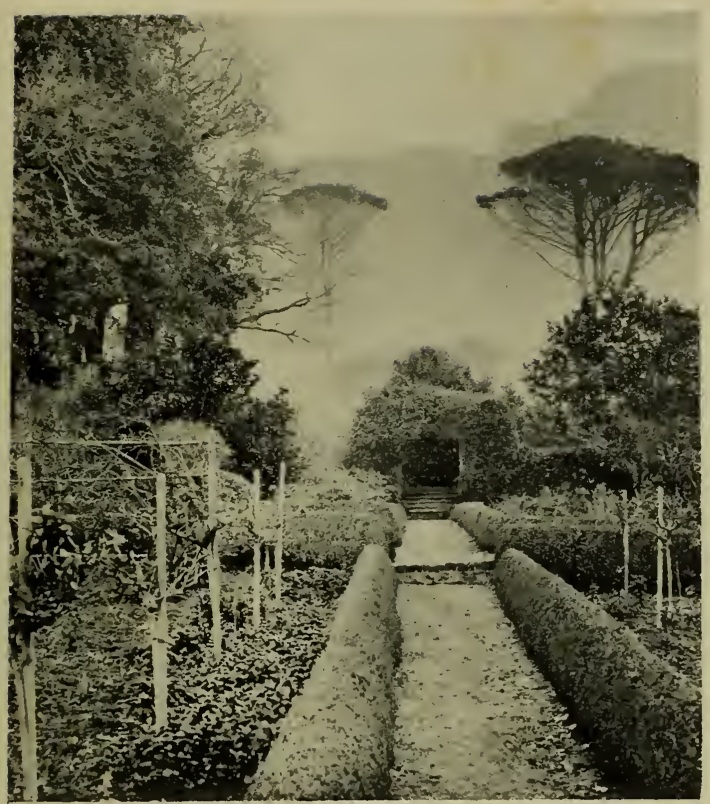

Dans le parc de "Groote-Schur"

ment une preuve, en prenant part, en juin ı10, comme délégué spécial du comité exécutif, à la remarquable conférence universelle missionnaire d'Edimbourg, qui a démontré par des faits la marche conquérante du christianisme dans le monde entier.

Le 13 juin, le lord-maire d'Edimbourg, entouré de ses conseillers, a donné une magnifique réception à laquelle cinq mille personnes avaient été invitées. Le lendemain, lord Balfour of Burleigh, président honoraire, a ouvert la conférence universelle missionnaire L'archevêque de Canterbury, qui officiait il y a quelques mois aux obsèques d'Edouard VII, a 
prononcé l'un des discours d'ouverture en souhaitant la bienvenue aux douze cents délégués, sans compter un nombre au moins triple de visiteurs, qui représentaient cent cinquante-neuf sociétés de missions évangéliques.

La Société des Missions de la Suisse romande avait délégué son secrétaire général, M. Grandjean, qu'accompagnaient M. Henri Junod missionnaire en Afrique et le pasteur Bugnion. La Société des Missions évangéliques de Paris était représentée par son directeur, M. Bœgner, et plusieurs autres délégués.

Divers journaux politiques influents ont suivi avec attention les délibérations de la conférence et, ainsi que l'a fait remarquer le "Daily News ", l'une de ses caractéristiques a été l'union dans un même sentiment de l'importance du but à atteindre; les chefs des. dénominations religieuses évangéliques les plus diverses, depuis les hauts dignitaires de l'Eglise anglicane jusqu'aux non-congrégationalistes, se sont tendus une main fraternelle.

Un grand nombre de laïques influents ont pris une part active à la conférence dont John Mott a rempli les fonctions de président effectif; parmi eux Sir Andrew Fraser, le gouverneur du Bengale; l'homme d'état, W.-H. Bryan, qui a été le concurrent de Taft aux dernières élections présidentielles des Etats-Unis, etc. Mais j'allais omettre de mentionner le $\mathrm{D}^{\mathrm{r}}$ Capen, de Boston, qui fut l'initiateur, au Canada et aux Etats-Unis, du "Laymen Movement " soit du travail qui a été fait récemment parmi 
les laïques, surtout hommes d'action et hommes d'affaires, pour les intéresser à l'œuvre missionnaire et avoir leur appui. Ce travail qui a donné de magnifiques résultats mériterait d'être étudié et suivi dans tous les pays. On a comparé la conférence universelle d'Edimbourg aux grands conseils œcuméniques du passé... avec cette différence, que jadis les débats en étaient âpres et souvent d'une grande violence; tandis qu'aujourd'hui, véritable trêve de Dieu, les membres de la conférence se sont réunis dans un esprit de bonne volonté et de bienveillance réciproques en vue des résultats pratiques à réaliser. J'ajouterai que plusieurs orateurs ont aussi parlé de l'œuvre internationale des Unions chrétiennes, soit de jeunes gens, soit de jeunes filles, en appréciant ces œuvres, non seulement au point de vue évangélique, mais comme "œuvre d'union" parmi les différentes églises.

Il était émouvant d'entendre des Africains, des Chinois, des Japonais, des Coréens, des Hindous eux-mêmes preuves vivantes de la puissance régénératrice des principes chrétiens, - exposer en termes excellents et souvent pathétiques, combien leurs patries respectives requéraient actuellement le secours et les lumières du christianisme.

Il ne faut pas se dissimuler que la tâche à accomplir est immense. Et pourtant que d'éclatantes victoires peuvent déja être enregistrées! L'autre jour, cinq cents jeunes étudiants de l'Université de Pékin ne se sont-ils pas engagés volontairement pour aider 
à l'évangélisation de leur propre pays? On sait le développement que l'œuvre missionnaire a pris en Chine ces dernières années, où l'on compte aujourd'hui trois mille neuf cents missionnaires évangéliques, qui, sans parler des stations secondaires, ont des champs d'action dans plus de cinq cents villes de l'immense empire du Milieu.

Nos souvenirs de la conférence mondiale de Tokio, 1907, et du centenaire missionnaire de Chine à Shanghaï sont encore bien vivants. Au Japon, le christianisme est non seulement implanté, mais naturalisé, ainsi qu'en font foi les quatre-cents églises japonaises évangéliques déjà organisées.

En 1876 , l'Afrique, sauf les colonies côtières, était le continent noir, inconnu, mystérieux; nous y comptons à l'heure qu'il est deux mille cinq cents missionnaires qui ont créé quatre mille écoles. Que dire du travail déjà accompli en Uganda, dans le district des Grands-Lacs, au Congo, sur le HautZambèze, au Basoutoland et dans nombre d'autres contrées africaines. Combien ces courageux pionniers de l'Evangile, les missionnaires dispersés dans les parties les plus reculées de la terre, ne devraient-ils pas être, plus encore qu'ils ne le sont, entourés de symphathie et aidés au point de vue matériel? Leur œuvre est civilisatrice dans l'acception la plus noble et la plus élevée de ce mot.

Afin d'être juste, il faudrait encore citer tous les services que de nombreux missionnaires ont rendus à la science, surtout à la géographie et à la philolo- 
gie, et dire combien, comme explorateurs, ils ont étendu le champ des connaissances humaines.

Les prochaines années constitueront, selon toutes probabilités, une époque décisive dans l'histoire de

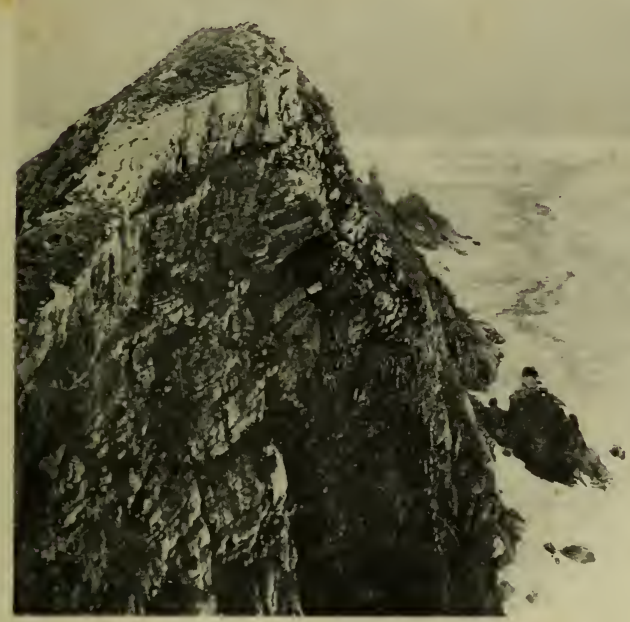

Cap de Bonne-Espérance

l'humanité. Il est probable qu'elles auront dans l'évolution de la race humaine, une importance plus grande que celle de bien des siècles dans le passé. Si elles sont bien employées elles pourront compter parmi les plus glorieuses de l'histoire du christianisme.

A notre retour au Cap, nous avons dû prendre la parole dans plusieurs meetings. En outre, Madame Bertrand a pu faire part à l'Union chrétienne des jeunes filles du Cap, comme cela avait été déjà le cas 
à Blœmfontein, de l'initiative qu'elle avait prise en fondant à Mafubé, dans l'East-Griqualand, une activité unioniste parmi les femmes noires.

Nous avons été invités à assister à la séance historique du Parlement qui a ratifié la Confédération des Etats de l'Afrique du Sud. Ainsi que l'a fait observer le gouverneur du Cap dans le discours prononcé à cette occasion, ce fait marquera comme l'un des plus importants de l'histoire du Sud-Africain.

En réunissant en une confédération la colonie du Cap, le Transvaal, l'Etat d'Orange et le Natal, les barrières douanières n'existeront plus dans ces quatre états. Sans parler de tous les autres avantages créés par ce nouvel ordre de choses, une ère économique nouvelle s'ouvre pour cette vaste contrée qui couvre une superficie de plus de sept cent cinquante mille kilomètres carrés.

L'ouverture du premier parlement de "l'Union» qui aura lieu le 4 novembre 1910 , sous la présidence du duc de Connaught, représentant le roi d'Angleterre, sera sans doute le prélude d'une ère de grande prospérité pour l'ensemble des Etats confédérés du Sud-Africain.

Ensuite le duc de Connaught se rendra aux chutes Victoria du Haut-Zambèze et il recevra, le I4 novembre, au settlement de Livingstone, Léwanika, roi des ba-Rotsi.

Il est peut-être intéressant de mentionner, que "Groote Schur ", le superbe domaine adossé aux flancs de la montagne de la Table, et créé par feu 
Cécil Rhodes, deviendra désormais la résidence du premier-ministre de la nouvelle Confédération, qu'il soit anglais ou boer.

La veille de notre départ, je suis invité au dîner du City Club, en l'honneur de l'ancien premierministre, le $\mathrm{D}^{\mathrm{r}} \mathrm{J}$ ameson, l'un des délégués choisis pour se rendre en Angleterre en vue de la ratification de la nouvelle Confédération des Etats du SudAfricain par les Chambres et le Roi. Nombre d'hommes intéressants prennent part à ce dîner historique, entre autres F.-W. Smith, le maire de la ville du Cap; Hon. E. Walton, l'ancien Trésoriergénéral; l'ancien ministre des Travaux publics, etc.

La table du banquet est gracieusement ornée de fleurs et de flots de rubans rouges, jaunes, blancs, bleus et verts; ce sont les couleurs des différents états qui formeront "l'Union of South-Africa".

Le maire de la ville du Cap et l'ancien premierministre prononcent des discours d'un esprit élevé, inspirés qu'ils sont par le plus chaud patriotisme; ils insistent sur les bienfaits que créera la nouvelle Confédération des Etats du Sud-Africain.

Le jour suivant nous nous embarquons à bord du "Kildonan Castle " pour notre retour en Europe. Le $\mathrm{D}^{\mathrm{r}}$ Jameson et Sir Charles Metcalf, l'ingénieur en chef de la ligne ferrée du Cap au Caire, se trouvent parmi les passagers : nous aurons avec eux plus d'une conversation intéressante. 



\section{Ou même भuteur:}

\section{AU PAYS DES BA-ROTSI - HAUT-ZAMBÈZE} VOYAGE D'EXPLORATION EN AFRIQUE et retour par les Chutes Victoria, le Matébéléland, le Transvaal, Natal, le Cap.

Ouvrage illustré de 105 gravures et de deux cartes.

$$
\text { PARIS - HACHETTE } 1908 \text { - Épuise }
$$

Traduction de l'ouvrage ci-dessus, par A. B. MIALL

\section{THE KINGDOM OF THE BAROTSI}

LONDON - T. FISHER-UNWIN, Paternoster Square, 1899 - (Épuisé)

\section{EN AFRIQUE AVEC LE MISSIONNAIRE COILLARD}

A travers l'Etat libre d'Orange, le Pays des ba-Souto, retour par la Côte orientale: Matébéléland, Mashonaland, Territoire de la Cie de Mozambique. Beira. Diégo-Suarez au N.-E. de Madagascar.

Ouvrage illustré de 38 gravures d'après les photographies de l'auteur. GENÈVE - CH. EGGIMANN \& Cie., Editeur. - 2e édition

ஆ QUELQUES NOTES SUR LES CONFÉRENCES DE TOKYO ET DE SCHANGHÄ̈

et voyage de retour par le Canada, les Montagnes rocheuses, les Chutes du Niagara, etc., avec illustrations d'apres photographies des auteurs.

GENÈVE 1907

N. B. n'a pas été mis en vente. 
PHOTOTYPIE ET IMPRESSION

Secheron - GENĖVE - (Suisse)

BELLEGARDE (Ain)

1911 



